\title{
A SEARCH FOR “DWARF” SEYFERT NUCLEI. VII. A CATALOG OF CENTRAL STELLAR VELOCITY DISPERSIONS OF NEARBY GALAXIES
}

\author{
Luis C. Ho ${ }^{1}$, Jenny E. Greene ${ }^{2,5}$, Alexei V. Filippenko ${ }^{3}$, and Wallace L. W. Sargent ${ }^{4}$ \\ ${ }^{1}$ The Observatories of the Carnegie Institution of Washington, 813 Santa Barbara Street, Pasadena, CA 91101, USA \\ ${ }^{2}$ Department of Astrophysical Sciences, Princeton University, Princeton, NJ, USA \\ ${ }^{3}$ Department of Astronomy, University of California, Berkeley, CA 94720-3411, USA \\ ${ }^{4}$ Palomar Observatory, California Institute of Technology, MS 105-24, Pasadena, CA 91125, USA \\ Received 2008 September 11; accepted 2009 May 7; published 2009 June 16
}

\begin{abstract}
We present new central stellar velocity dispersion measurements for 428 galaxies in the Palomar spectroscopic survey of bright, northern galaxies. Of these, 142 have no previously published measurements, most being relatively late-type systems with low velocity dispersions $\left(\lesssim 100 \mathrm{~km} \mathrm{~s}^{-1}\right)$. We provide updates to a number of literature dispersions with large uncertainties. Our measurements are based on a direct pixel-fitting technique that can accommodate composite stellar populations by calculating an optimal linear combination of input stellar templates. The original Palomar survey data were taken under conditions that are not ideally suited for deriving stellar velocity dispersions for galaxies with a wide range of Hubble types. We describe an effective strategy to circumvent this complication and demonstrate that we can still obtain reliable velocity dispersions for this sample of well-studied nearby galaxies.
\end{abstract}

Key words: galaxies: active - galaxies: kinematics and dynamics - galaxies: nuclei - galaxies: Seyfert - galaxies: starburst - surveys

Online-only material: machine-readable table

\section{INTRODUCTION}

The stellar velocity dispersion $\left(\sigma_{\star}\right)$ of the central regions of galaxies is a parameter of considerable importance for a variety of extragalactic investigations. Since the early pioneering work of Burbidge et al. (1961) and Minkowski (1962), many techniques have been developed for measuring $\sigma_{\star}$ (e.g., Morton \& Chevalier 1972; Richstone \& Sargent 1972; Simkin 1974; Sargent et al. 1977; Tonry \& Davis 1979; Bender 1990; Rix \& White 1992; van der Marel \& Franx 1993; Statler 1995; Barth et al. 2002; Cappellari \& Emsellem 2004). Given the extensive body of observational material on $\sigma_{\star}$ for nearby galaxies, a number of catalogs have been compiled to consolidate the data. The most widely used of these are the catalog of Whitmore et al. (1985), which was updated by McElroy (1995), and of Prugniel et al. (1998), which is continuously updated and is available through the electronic database HyperLeda (Paturel et al. 2003). ${ }^{6}$

The vast majority of the published measurements of $\sigma_{\star}$ pertain to early-type galaxies, largely giant ellipticals and S0s. Significantly less data are available for galaxies along the spiral sequence, and those that have been published often show marked disagreement from study to study, as can be seen from perusal of the data tabulated in the above-mentioned catalogs. It is disconcerting that many of the highly discrepant entries are, in fact, associated with nearby, bright, well-studied galaxies. The scatter in the published values of $\sigma_{\star}$ can be blamed, at least in part, on the inherent heterogeneity of combining many disparate sources, which often employ different telescopes, detectors, apertures, observing strategies, and analysis techniques. The above-cited catalogs attempt to homogenize the final compilations by scaling the individual literature sources to a set of "standard" galaxies measured through a roughly constant aperture size $\left(2^{\prime \prime} \times 4^{\prime \prime}\right)$.

\footnotetext{
5 Hubble Fellow, Princeton-Carnegie Fellow.

6 http://leda.univ-lyon1.fr/
}

Notwithstanding these efforts, there is considerable motivation for assembling an independent, homogeneous, internally consistent set of new measurements, especially if the data cover a large sample of galaxies representing a wide range of Hubble types. A number of previous studies have been carried out with this goal in mind, mostly focused on relatively early-type galaxies (e.g., Davies et al. 1987; Bernardi et al. 2003). Our present paper adds to this effort using data taken as part of the Palomar spectroscopic survey of nearby galaxies. During the course of an extensive investigation primarily aimed at characterizing the nature of nuclear activity in nearby galaxies, we collected high-quality, moderate-resolution, long-slit optical spectra of the central regions of 486 bright, northern galaxies. The survey was conducted during the period 1984-1990; technical details of the survey and presentation of various data products and science results can be found in earlier papers in this series (Filippenko \& Sargent 1985; Ho et al. 1995, 1997a, 1997b, 1997c, 1997d, 1997e, 2003). This contribution focuses on central stellar velocity dispersions extracted from the survey.

\section{THE SURVEY}

A full description of the Palomar survey is given by Ho et al. $(1995,1997 a)$. Here we mention only a few pertinent details. The survey covers a nearly complete, magnitude-limited sample of 486 galaxies from the Revised Shapley-Ames Catalog (Sandage \& Tammann 1981) that satisfies $B_{T} \leqslant 12.5 \mathrm{mag}$ and $\delta>0^{\circ}$. The spectra were acquired using the Double Spectrograph (Oke \& Gunn 1982) mounted at the Cassegrain focus of the Hale $5 \mathrm{~m}$ telescope at Palomar Observatory. A 2" wide slit was used for most of the survey. The spectra simultaneously cover the regions $\sim 4230-5110 \AA$ and $\sim 6210-6860 \AA$. The average full width at half-maximum intensity (FWHM) spectral resolutions on the blue and red sides, as determined from comparison-lamp emission lines, are approximately $4.2 \AA$ and $2.2 \AA$, respectively. These correspond to velocity resolutions, expressed as a Gaussian dispersion, of $\sigma_{\text {inst }}=118$ and $42 \mathrm{~km}$ 

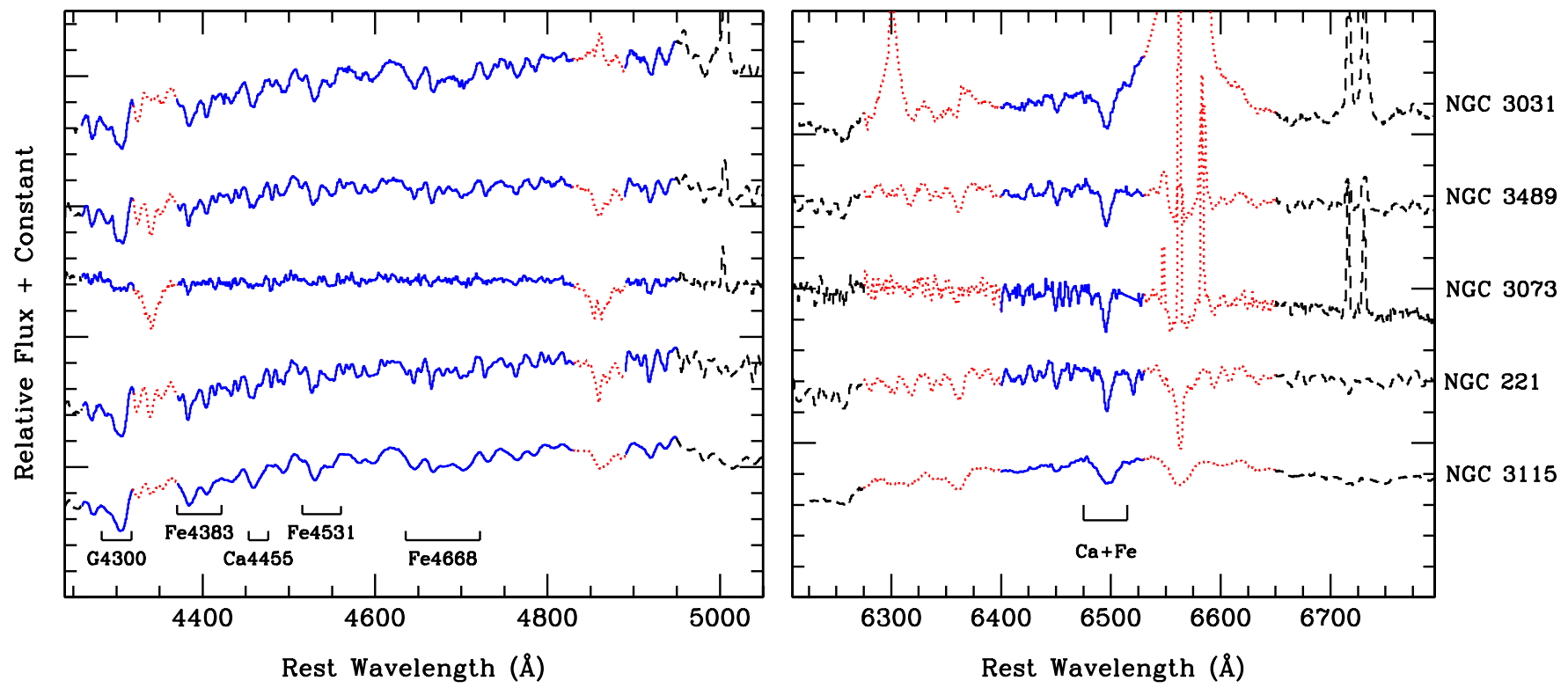

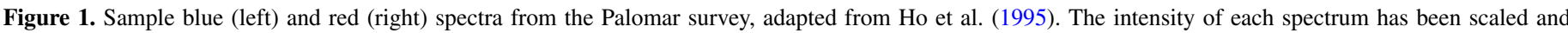

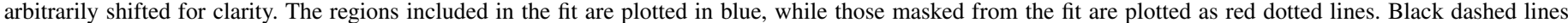
denote regions outside of the fitting window. The stellar metal-line indices defined by Ho et al. (1997a) are labeled on the bottom of each panel.

$\mathrm{s}^{-1}$ at $4500 \AA$ and $6500 \AA$, respectively. (About $10 \%$ of the blue spectra were acquired in a slightly higher resolution mode with $\sigma_{\text {inst }}=74 \mathrm{~km} \mathrm{~s}^{-1}$.) The spectra analyzed in this paper are the same as those reported in the spectral atlas of Ho et al. (1995); they were extracted from a rectangular aperture of size $2^{\prime \prime} \times 4^{\prime \prime}$, which is roughly equivalent to linear dimensions of $170 \mathrm{pc} \times$ $350 \mathrm{pc}$ for a median distance of 17.9 Mpc (Ho et al. 1997a).

\section{VELOCITY DISPERSIONS}

\subsection{Method}

Our velocity dispersion measurements are based on the direct pixel-fitting method, which, as described by a number of authors (e.g., Rix \& White 1992; van der Marel 1994; Kelson et al. 2000; Barth et al. 2002), has many of advantages compared to more traditional methods based on Fourier or cross-correlation techniques. The Palomar survey has several characteristics that pose special challenges for measuring accurate stellar velocity dispersions. First, the majority of the survey galaxies contain emission lines from active galactic nuclei (AGNs), often strong and of substantial velocity width, presenting a significant source of contamination for the stellar absorption features. Second, the spectral coverage of the survey was optimized for obtaining emission-line diagnostics and not for velocity dispersion measurements. Finally, the survey covers a very broad range of Hubble types, from dwarf irregulars to giant ellipticals. Galaxies with a wide range of stellar populations are especially susceptible to template mismatch. We use a modified version of the direct pixel-fitting code developed by Greene \& Ho (2006). In brief, a nonlinear Levenberg-Marquardt minimization algorithm is used to compare the observed galaxy spectrum with a model spectrum $M(\lambda)$, which is assumed to be the convolution of a stellar template spectrum, $T(\lambda)$, and a line-of-sight velocity broadening function approximated as a Gaussian, $G(\lambda)$ :

$$
M(\lambda)=P(\lambda)\{[T(\lambda) \otimes G(\lambda)]+C(\lambda)\}
$$

Here, $C(\lambda)$ is an additive term to dilute the stellar features. It can be a power-law function to represent an AGN continuum, if present, or any other smooth component such as the featureless continuum from hot stars. For many of our later-type galaxies, adding a simple $f_{\lambda}=$ constant term effectively mimics the continuum dilution of the metal lines by intermediate-age (A and early-F type) stars in the composite stellar population. The multiplicative factor $P(\lambda)$, typically chosen to be a third-order Legendre polynomial, accounts for large-scale mismatches in the continuum shapes of the galaxy and template star(s), which can arise from internal reddening in the galaxy, stellar population differences, and possible residual calibration errors.

An important improvement over the original code of Greene \& Ho is that $T(\lambda)$, rather than being a single star, can be an optimal linear combination of several stars determined through a nonlinear least-squares fit. In the case of later-type spirals, especially, this modification provides a much better fit for their composite stellar populations, as well as a more robust determination of the final velocity dispersion of the galaxy because the intrinsic widths of the template stars vary with spectral type. Our approach of using a mixture of template stars is similar to those employed by several previous studies, including Rix \& White (1992) and Cappellari \& Emsellem (2004).

\subsection{Fitting Regions}

The blue setup just misses $\mathrm{Mg}$ I $\lambda 5175$ ("Mg $b$ "), the feature most commonly used to derive velocity dispersions in the visible part of the spectrum. Nevertheless, the blue spectra contain a significant number of relatively strong metal-line features, including the $\mathrm{G}$ band at $4300 \AA$, a calcium feature at $4455 \AA$, and iron features at 4383, 4531, and $4668 \AA$ (Figure 1, left; see Table 7 in Ho et al. (1997a) for definitions of these stellar absorption-line indices). These metal-line features can be used to derive stellar velocity dispersions, so long as they are strong enough in the integrated spectrum. For the blue spectra we fit the region 4260-4950 $\AA$; the blue end is chosen to include the $G$ band, while the red end avoids the [O III] $\lambda \lambda 4959,5007$ emission 
lines. We mask the regions containing $\mathrm{H} \gamma(4320-4370 \AA)$ and $\mathrm{H} \beta$ (4830-4890 $\AA$ ). In some strong emission-line objects, it is necessary to mask a small region around He II $\lambda 4686$.

In practice, the above procedure works well for galaxies with a stellar population dominated by stars of spectral type mid-F and later, but not for those with younger populations. As Figure 1 illustrates, the spectrum of NGC 3073 contains mostly light from stars of type A and early-F, and the metal-line features, although clearly present in this spectrum of fairly high signal-to-noise ratio $(\mathrm{S} / \mathrm{N})$, are significantly diluted by the blue continuum of the hotter stars. Spectra like that of NGC 3073 (which, curiously, is an S0 galaxy) typically characterize many of the later-type spirals in the survey. The moderate resolution of the blue spectra presents another severe limitation. Even for galaxies where the blue metal-line features are strong and unambiguously detected (e.g., NGC 221 and NGC 3489 in Figure 1), the derived velocity dispersions may be subject to large systematic uncertainties if the true dispersions are near or below the native spectral resolution of $\sigma_{\text {inst }} \approx 120 \mathrm{~km} \mathrm{~s}^{-1}$. For example, according to the literature NGC 221 and NGC 3489 have $\sigma=72$ and $112 \mathrm{~km}$ $\mathrm{s}^{-1}$, respectively.

The red spectra, with $\sigma_{\text {inst }} \approx 40 \mathrm{~km} \mathrm{~s}^{-1}$, provide crucial relief to the many galaxies in the survey that suffer from insufficient resolution in the blue. Unfortunately, very few strong, uncontaminated stellar features exist in the spectral coverage of our red setup, which is dominated almost entirely by strong emission lines ([O I] $\lambda \lambda 6300,6363$, [N II] $\lambda \lambda 6548,6583$, $\mathrm{H} \alpha$, and [S II] $\lambda \lambda 6716,6731)$. One glimmer of hope lies with the $\mathrm{Ca}+\mathrm{Fe}$ feature at $6495 \AA$. To the best of our knowledge, this little-known feature has never been used explicitly for kinematical measurements in galaxies, although it has played a role in other contexts such as the determination of radial-velocity curves for the secondary stars in black hole X-ray binaries (e.g., Filippenko et al. 1995, 1997). We will show that it plays a central role in our survey.

As Figure 1 (right) illustrates, the $\mathrm{Ca}+\mathrm{Fe}$ feature, lying just blueward of the $\mathrm{H} \alpha+[\mathrm{N} \mathrm{II}]$ complex, is fairly well isolated, even in objects with prominent, broad $\mathrm{H} \alpha$ emission (e.g., NGC 3031). Importantly, it is moderately strong in nearly all galaxies, even those whose blue spectra are hopeless diluted by A and F-type stars (e.g., NGC 3073). Using the measurements published by $\mathrm{Ho}$ et al. (1997a, Table 9), we find that $\mathrm{Ca}+\mathrm{Fe}$ was reliably detected in 438 out of the 486 galaxies in the Palomar survey $(90 \%)$, with an average equivalent width of $\langle\mathrm{W}(\mathrm{Ca}+\mathrm{Fe})\rangle=0.9 \AA$. There is, at most, a factor of 2 variation in line strength from one extreme end of the Hubble sequence to the other. Among ellipticals and S0s (morphological type index $-6<T<0$; de Vaucouleurs et al. 1991), $\langle\mathrm{W}(\mathrm{Ca}+\mathrm{Fe})\rangle=$ $1.2 \AA$, to be compared with $\langle\mathrm{W}(\mathrm{Ca}+\mathrm{Fe})\rangle=0.6 \AA$ for $\mathrm{Sc}-\mathrm{Sdm}$ spirals (morphological type index $5<T<9$ ).

After some experimentation, we find that the most stable fitting region for the red setup is 6400-6530 A (Figure 1, right). The blue limit provides as much leverage as possible to define the continuum level without colliding with $\left[\mathrm{O}_{\mathrm{I}}\right] \lambda 6363$, and the red limit abuts $[\mathrm{N}$ II] $\lambda 6548$. In a few objects with very strong, broad $\mathrm{H} \alpha$ emission, we had to curtail the red limit to $6510 \AA$; in these cases, it was often also helpful to increase the order of the polynomial factor (to $\sim 5-6$ ) to better trace the steeply rising gradient of the blue wing of the $\mathrm{H} \alpha$ emission line.

\subsection{Template Stars}

In addition to spectrophotometric standard stars, during the course of the survey we usually also took nightly observations of at least one late-type giant star to be used as a velocity template. Velocity standards were not observed in a small number of observing runs; this affected 50 galaxies, or roughly $10 \%$ of the survey. Because measuring velocity dispersions was not a top priority for the original survey, neither the number of stars nor their range of spectral types was chosen optimally. In some of the runs, only a single velocity template was observed, and at most there were two.

The limitations of the Palomar template stars compel us to explore an alternative calibration strategy. We use as our primary source of templates the library of Coudé-feed stellar spectra published by Valdes et al. (2004). This tremendously useful database contains high-S/N spectra of 1273 stars of essentially all spectral types, covering 3460 to $9464 \AA$. The spectral resolution of the library, FWHM $\approx 1 \AA$, is significantly higher than that of either the blue or red Palomar spectra. Thus, the Valdes stars can be used as velocity templates for the Palomar galaxies, after accounting for the differential instrumental broadening between the two data sets.

The Valdes library also gives us an extensive selection of stars of different spectral types for our optimal fit. Through experimentation, we find that in general a set of four starsspectral types F6 III, G8 III, K0 III, and K3 III-suffices to account for the stellar population mixture of almost all galaxies in our sample. We give preference to stars of nearsolar metallicity to try to approximate the conditions in galactic bulges. Although type-A and early-F stars clearly exist in some galaxies, in practice they do not need to be included because our fitting regions deliberately avoid the Balmer absorption lines (Figure 1) and the continuum dilution term $(C(\lambda)$ in Equation (1)) effectively mimics the hot continuum of these stars.

\subsection{Fitting Results}

Figure 2 gives examples of some typical fits. The top spectrum is that of the red giant (K0 III) star HD 107328, shown to help guide the eye to identify the stellar features. Subsequent spectra illustrate galaxies with a wide range in emission-line strengths and velocity dispersions. The original galaxy spectrum is plotted as black histograms; the best-fitting, optimally weighted, broadened velocity template is plotted as a thin blue line; and the masked regions are plotted as a red dotted line. Using a set of just four stars, we can usually achieve quite good fits, with formal statistical errors on the velocity dispersions in the range of 5\%-10\%. The results are also quite robust with respect to the choice of template stars; interchanging different stars of the same spectral type and similar metallicity affects the final dispersions at the level of $1 \%$ or less. In most objects, the largest fraction of the light comes, not surprisingly, from $\mathrm{K}$ giants. The Fe $\lambda 4668$ feature, in particular, is very sensitive to K1 IIIK3 III giant stars, which significantly improve the fit over the region 4600-4800 $\AA$ (Figure 3). Our fitting region for the red setup, especially the $\mathrm{Ca}+\mathrm{Fe} \lambda 6495$ feature, is also very sensitive to K1 III-K3 III giants (Figure 4). The vast majority of the galaxies, however, including many bulge-dominated systems, require some contribution from $\mathrm{G}$ and even F-type stars.

To translate the Valdes-based dispersions onto the Palomar system, we subtract in quadrature the relative resolution difference between the Valdes and Palomar systems. Assuming the nominal instrumental resolutions of the two data sets, the resolution correction for the blue side is $\sigma_{c}=114.8 \pm 5.8 \mathrm{~km}$ $\mathrm{s}^{-1}\left(68.4 \pm 7.1 \mathrm{~km} \mathrm{~s}^{-1}\right.$ for the higher resolution mode), while that for the red side is $\sigma_{c}=37.4 \pm 7.5 \mathrm{~km} \mathrm{~s}^{-1}$, where the 


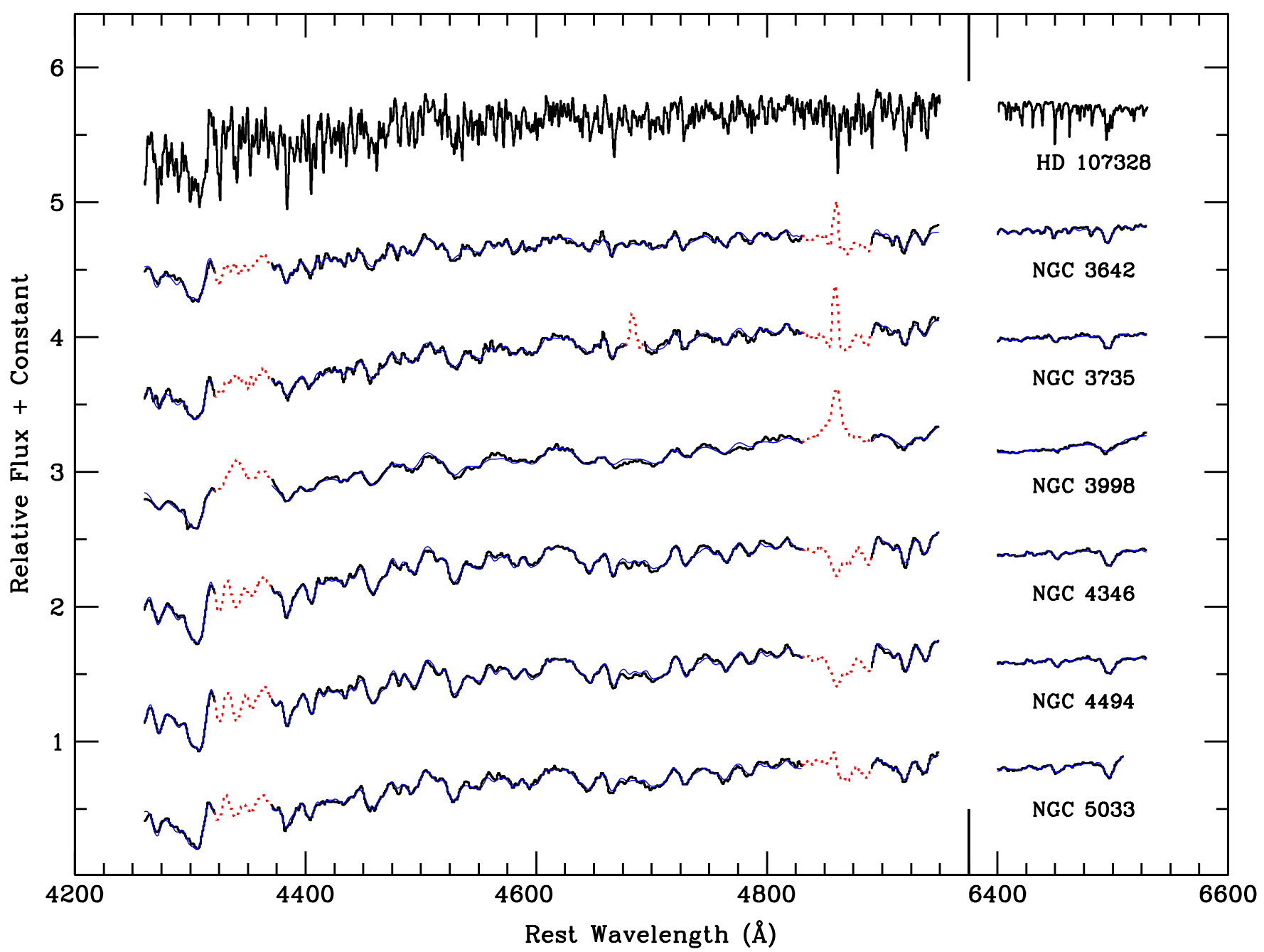

Figure 2. Sample fits for a representative set of galaxies. The top spectrum is that of the K0 III star HD 107328 from the Valdes et al. (2004) stellar library. For each galaxy, the original data are plotted as black histograms. The best-fitting model constructed from an optimal combination of broadened stellar templates is plotted as a thin blue curve. The regions excluded from the fit are marked as red dotted lines. The intensity of each spectrum has been scaled and arbitrarily shifted for clarity.

error bar represents the rms scatter of the night-to-night variations of the Palomar instrumental resolution. The validity of this simple approach can be verified empirically by comparing the corrected dispersions with published values. Among the 223 galaxies with velocity dispersions derived in the blue, 189 have literature measurements; of the 422 dispersions measured in the red, 283 have literature values. As illustrated in Figure 5, the adopted resolution corrections yield reasonably satisfactory agreement between our dispersion measurements and the literature values, particularly in the regime when the dispersions are well resolved ( $\sigma \gtrsim \sigma_{\text {inst }}$; solid points). On the blue side (Figure 5(a)), for $\sigma \gtrsim \sigma_{\text {inst }} \approx 120 \mathrm{~km} \mathrm{~s}^{-1},\left\langle\sigma_{\text {blue }}-\sigma_{\text {Literature }}\right\rangle=$ $1.2 \mathrm{~km} \mathrm{~s}^{-1}$ with an rms scatter of $25.3 \mathrm{~km} \mathrm{~s}^{-1}$. The red side delivers useful measurements down to $\sigma \approx \frac{1}{2} \sigma_{\text {inst }} \approx 20 \mathrm{~km} \mathrm{~s}^{-1}$ (Figure 5(b)). Over the entire velocity range, $\left\langle\sigma_{\text {red }}-\sigma_{\text {Literature }}\right\rangle=$ $3.0 \mathrm{~km} \mathrm{~s}^{-1}$ with an rms scatter of $28.3 \mathrm{~km} \mathrm{~s}^{-1}$. There is no perceptible systematic bias, provided that the optimal fit excludes the K3 III star, as explained below.

Our initial fits for the red-side spectra, which include the full complement of four template stars (F6 III to K3 III), revealed a puzzling systematic trend. Whereas the fits for low$\sigma$ galaxies yield dispersions that, after resolution correction, agree reasonably well with literature values, objects with $\sigma \gtrsim$ $150-200 \mathrm{~km} \mathrm{~s}^{-1}$ show a net systematic offset toward larger velocities, by roughly $+30 \mathrm{~km} \mathrm{~s}^{-1}$. We believe that this effect arises from template mismatch. As shown in Figure 4, in small, low-luminosity bulges, such as that in the Sc galaxy NGC 3631, the red absorption features, especially $\mathrm{Ca}+\mathrm{Fe}$, are nearly equally well fit by template stars of spectral type G8 III, K0 III, or K3 III. In stark contrast, NGC 3115, a luminous S0 galaxy with a substantial bulge, clearly singles out the K3 III star as the preferred template, which then contributes most of the weight to the optimal fit. (We have verified that $\mathrm{K} 1 \mathrm{III}$ and $\mathrm{K} 2 \mathrm{III}$ templates give almost equally good fits as the K3 III template.) Why? This is because the $\mathrm{Ca}+\mathrm{Fe}$ feature is strongest in high- $\sigma$ galaxies and in late-type giants. Within the Palomar galaxy sample, the strength of the $\mathrm{Ca}+\mathrm{Fe}$ feature increases roughly with velocity dispersion, albeit with significant scatter. Dividing the sample into two, galaxies with $\sigma<150 \mathrm{~km} \mathrm{~s}^{-1}$ have $\langle\mathrm{W}(\mathrm{Ca}+\mathrm{Fe})\rangle=0.87 \AA$, to be compared with $\langle\mathrm{W}(\mathrm{Ca}+\mathrm{Fe})\rangle=1.23 \AA$ for galaxies with $\sigma \geqslant$ $150 \mathrm{~km} \mathrm{~s}^{-1}$. At the same time, the strength of the $\mathrm{Ca}+\mathrm{Fe}$ feature in stars increases toward later spectral types. To demonstrate this, we measured the $\mathrm{Ca}+\mathrm{Fe}$ feature for individual stars in the Valdes library, using the index definition given in Ho et al. (1997a). Choosing 15 stars of roughly similar metallicities for each spectral type, we find $\langle\mathrm{W}(\mathrm{Ca}+\mathrm{Fe})\rangle=0.79,0.99,1.17$, 1.35 , and $1.53 \AA$ for G8 III, K0 III, K1 III, K2 III, and K3 III, respectively. Galaxies with $\sigma \geqslant 150 \mathrm{~km} \mathrm{~s}^{-1}$ have $\mathrm{Ca}+\mathrm{Fe}$ strengths very similar to those of K1 III-K3 III stars, and 

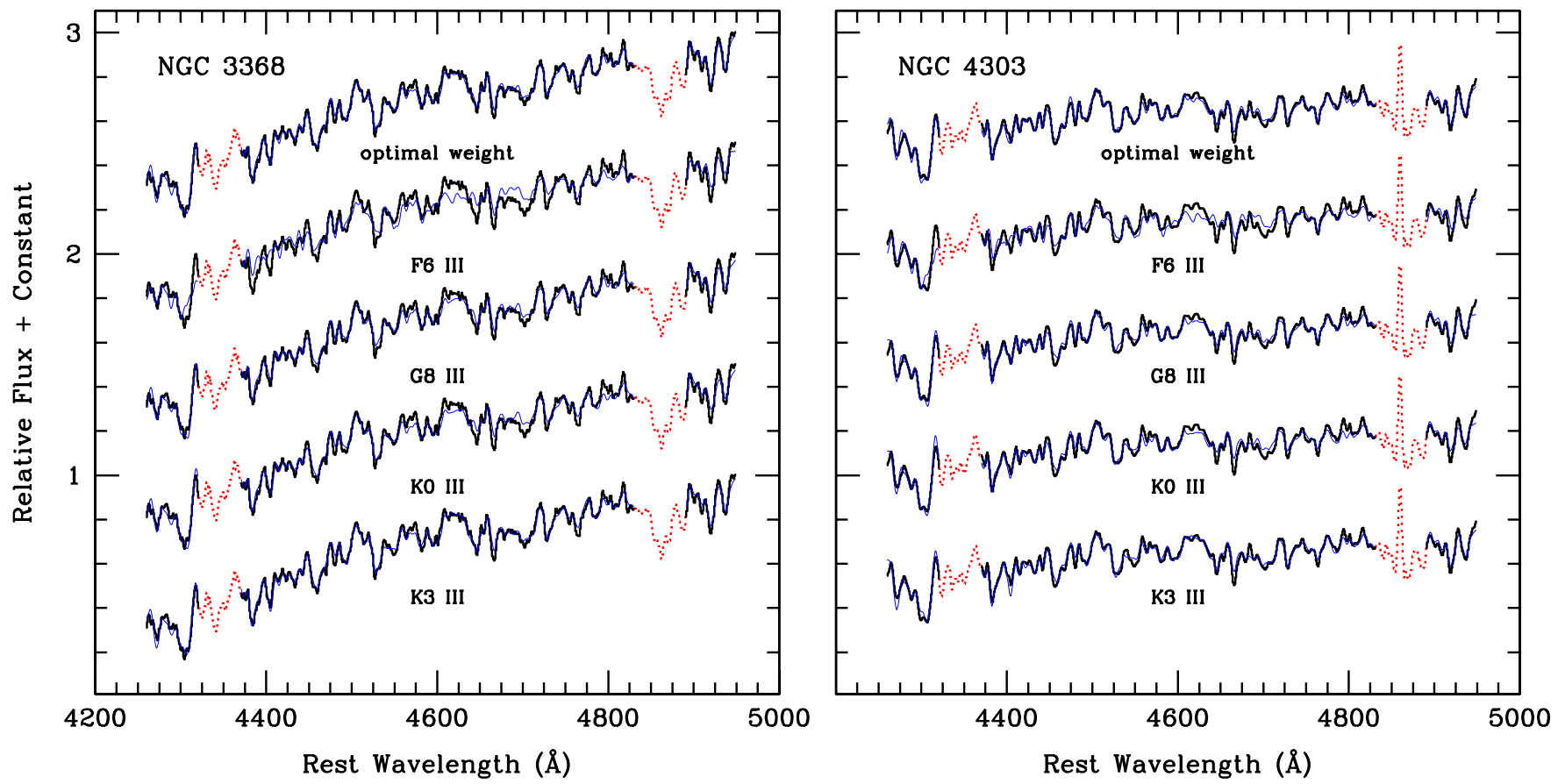

Figure 3. Sample fits for NGC 3368 and NGC 4303 in the blue spectral region. The top spectrum shows the optimally weighted fit, followed by fits using single stars of spectral type F6 III, G8 III, K0 III, and K3 III. The original data are plotted as black histograms, the fits are plotted as blue curves, and the regions excluded from the fit are plotted as red dotted lines. The intensity of each spectrum has been scaled and arbitrarily shifted for clarity.
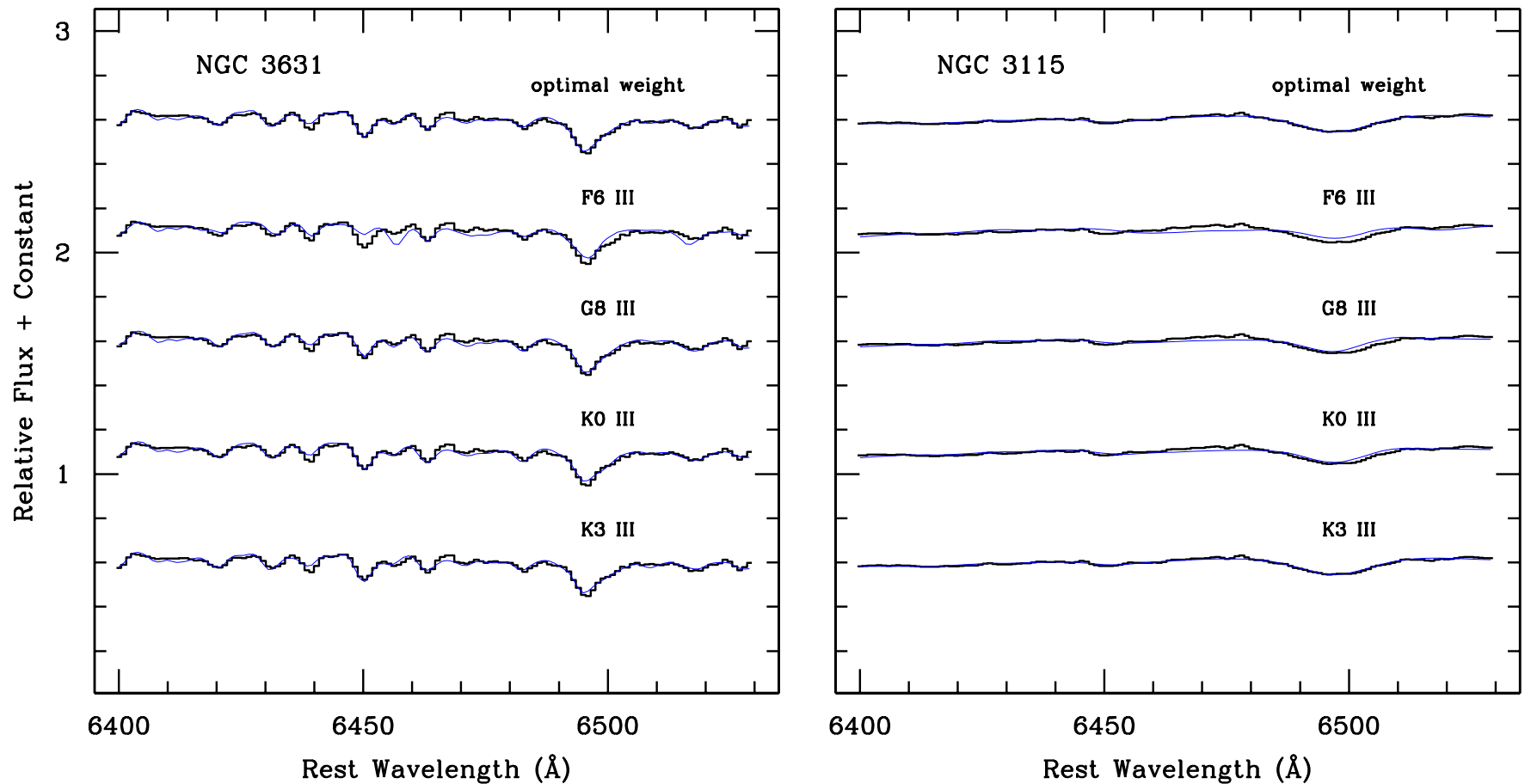

Figure 4. Sample fits for NGC 3631 and NGC 3115 in the red spectral region. The top spectrum shows the optimally weighted fit, followed by fits using single stars of spectral type F6 III, G8 III, K0 III, and K3 III. The original data are plotted as black histograms, and the fits are plotted as blue curves. The intensity of each spectrum has been scaled and arbitrarily shifted for clarity.

thus it is not surprising that an optimal fit would give these stars greatest weight. A bias in the derived velocity dispersion for high- $\sigma$ galaxies arises if in these systems their $\mathrm{Ca}+\mathrm{Fe}$ feature is boosted because of an abundance enhancement. We speculate that the culprit is $\mathrm{Ca}$. As an $\alpha$ element, Ca may be enhanced similarly as $\mathrm{Mg}$ in early-type galaxies (Prochaska et al. 2005; but see Graves et al. 2007). In such a situation, the apparently good match with the K1 III-K3 III templates is only an artifact of their mutually strong $\mathrm{Ca}+\mathrm{Fe}$ feature. Since such late-type giants have very narrow intrinsic line widths, the inferred velocity dispersion would be overestimated, thus leading to the observed bias. To bypass this complication, we removed the K3 III giant from the optimal fit of the red-side spectra. 

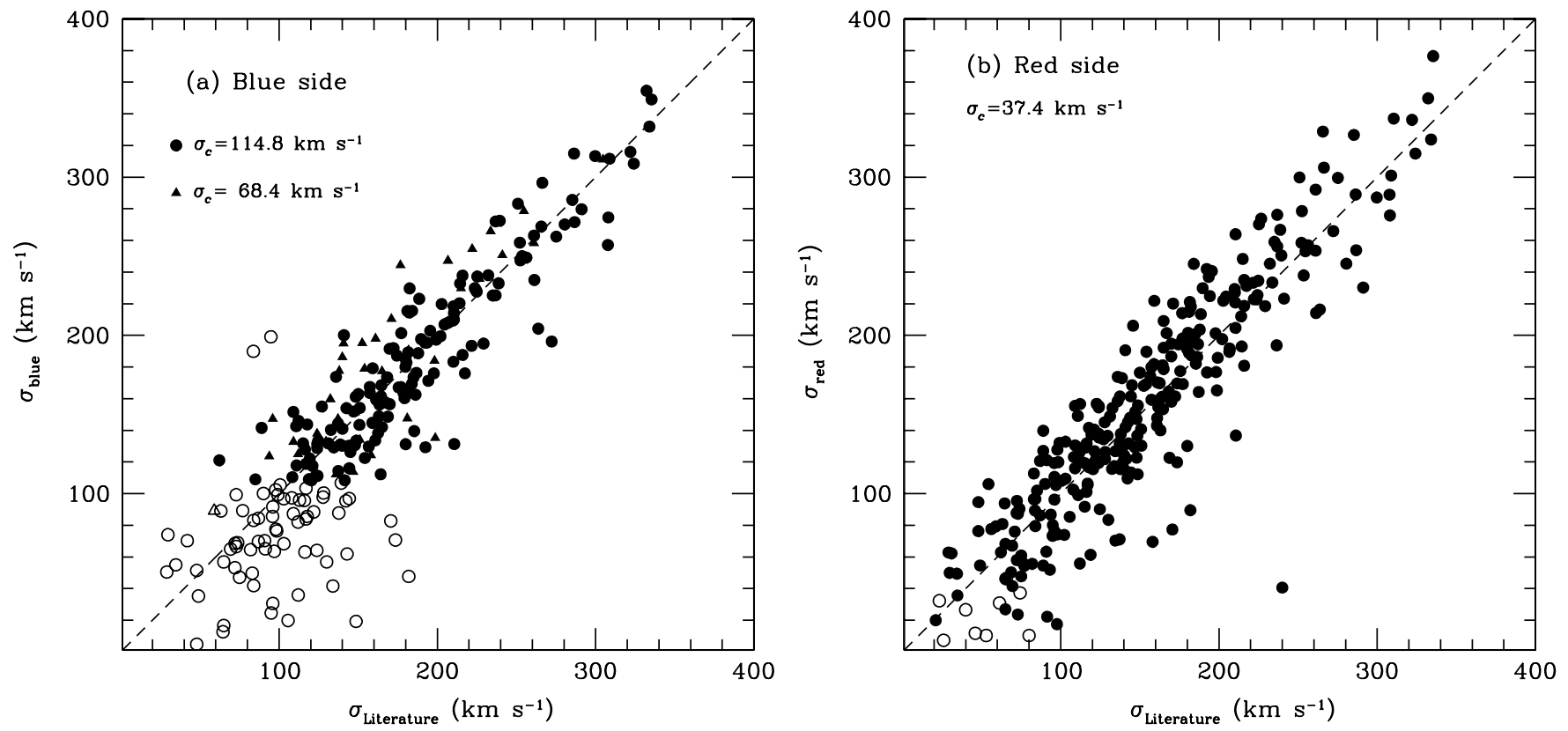

Figure 5. Comparison between velocity dispersions published in the literature with velocity dispersions derived using an optimal combination of Valdes template stars for the (a) blue side and (b) red side, corrected for the relative resolution difference $\left(\sigma_{c}\right)$ between the Valdes and Palomar systems (see Section 3.3). Open symbols mark objects that are poorly resolved. The dashed diagonal line denotes equality.

For each galaxy, we compute a final velocity dispersion as the average of the blue-side and red-side dispersions, weighted by their respective error bars. The error bars reflect the quadrature sum of the formal statistical uncertainty from the optimal fit and the rms scatter of the resolution correction, which is dominated by the uncertainty in the original instrumental resolution of the Palomar spectra. Among the 428 galaxies with new velocity dispersion measurements, 286 have published literature values. Comparison between the objects in common (Figure 6) shows very good consistency. Over the entire range in velocities, $\left\langle\sigma_{\text {final }}-\sigma_{\text {Literature }}\right\rangle=3.0 \mathrm{~km} \mathrm{~s}^{-1}$. The scatter is still quite large (rms $28.3 \mathrm{~km} \mathrm{~s}^{-1}$ ), but its magnitude is consistent with that found by Barth et al. (2002) based on a smaller sample of $\sim 30$ galaxies with high-quality velocity dispersion measurements.

There are several notable outliers in Figure 6, for which the literature values are larger than ours by more than $\sim 80 \mathrm{~km} \mathrm{~s}^{-1}$. The most extreme case is NGC 520, for which HyperLeda reports $\sigma=240 \pm 25 \mathrm{~km} \mathrm{~s}^{-1}$ whereas we determine $\sigma=40.6 \pm 8.9 \mathrm{~km} \mathrm{~s}^{-1}$. This is a complex, interacting galaxy (Arp 157), and the HyperLeda value of $\sigma=240 \mathrm{~km} \mathrm{~s}^{-1}$ pertains to the "southeast-northwest" component, not the primary nucleus of the "east-west" component (using the naming convention of Stanford \& Balcells 1990a). The Palomar spectrum was centered on the position of the primary nucleus. From visual inspection of the plots published by Stanford \& Balcells (1990a, 1990b), it appears that the published dispersion of the primary nucleus should be $\sigma \approx 100 \pm 25 \mathrm{~km} \mathrm{~s}^{-1}$. (We thank the referee for making this estimate for us, with which we agree.) The velocity dispersion for NGC 2967 (prior to homogenization), $200 \pm 27 \mathrm{~km} \mathrm{~s}^{-1}$, seems suspiciously high for an Sc galaxy; according to HyperLeda, it derives from an unpublished 1984 measurement by B. C. Whitmore and E. Malumuth. The same applies to the Sc galaxy NGC 4647, for which HyperLeda lists $\sigma=98 \pm 39 \mathrm{~km} \mathrm{~s}^{-1}$. Finally, we note that the literature values of both NGC $3628\left(\sigma=171 \pm 71 \mathrm{~km} \mathrm{~s}^{-1}\right)$ and NGC 5364 $\left(\sigma=91 \pm 52 \mathrm{~km} \mathrm{~s}^{-1}\right)$ have exceptionally large error bars. If we

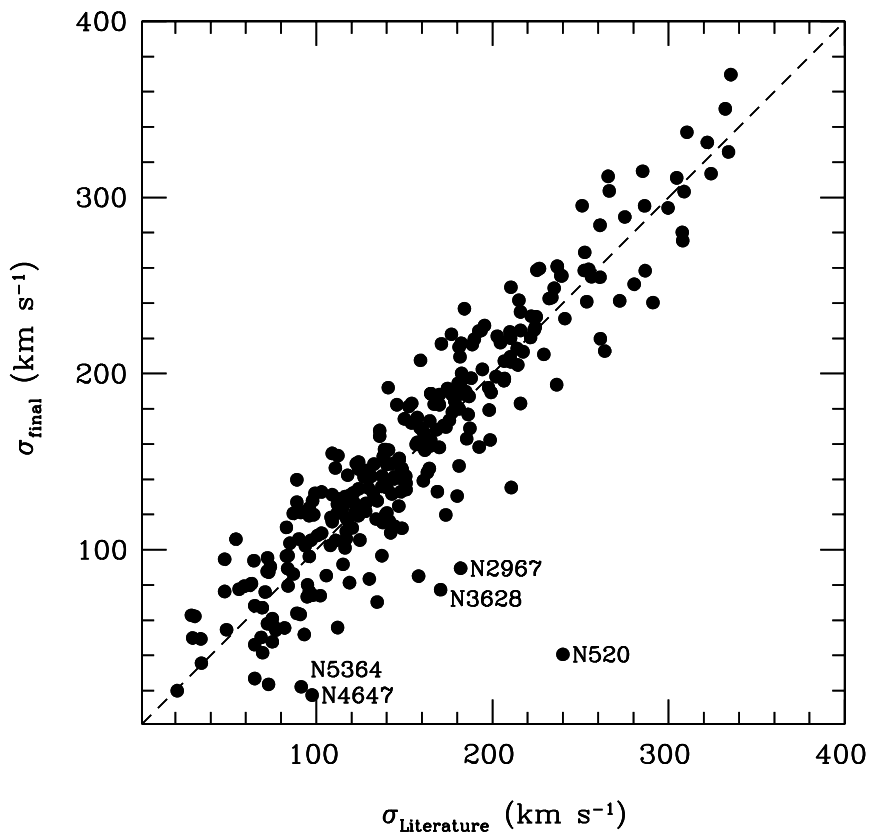

Figure 6. Comparison of final velocity dispersions with literature values. The dashed diagonal line denotes equality. Several prominent outliers are labeled (see Section 3.4).

exclude these five outliers from our sample, $\left\langle\sigma_{\text {final }}-\sigma_{\text {Literature }}\right\rangle=$ $2.7 \mathrm{~km} \mathrm{~s}^{-1}$, and the scatter reduces to $23.6 \mathrm{~km} \mathrm{~s}^{-1}$.

\section{THE CATALOG}

The final results are presented in Table 1. For each galaxy, we list the literature value of the central stellar velocity dispersion, if available, followed by the dispersions derived from the blue $\left(\sigma_{\text {blue }}\right)$ and red $\left(\sigma_{\text {red }}\right)$ Palomar spectra, the final value $\left(\sigma_{\text {final }}\right)$ obtained from the weighted average of $\sigma_{\text {blue }}$ and $\sigma_{\text {red }}$, and lastly 
Table 1

Catalog of Stellar Velocity Dispersions

\begin{tabular}{|c|c|c|c|c|c|c|c|}
\hline \multirow[t]{2}{*}{ Galaxy } & \multicolumn{2}{|c|}{ Literature } & \multicolumn{4}{|c|}{ Palomar } & \multirow{2}{*}{$\begin{array}{c}\text { Adopted } \\
\sigma \\
\left(\mathrm{km} \mathrm{s}^{-1}\right)\end{array}$} \\
\hline & $\begin{array}{c}\sigma \\
\left(\mathrm{km} \mathrm{s}^{-1}\right) \\
\end{array}$ & Reference & $\begin{array}{c}\begin{array}{c}\sigma_{\text {blue }} \\
\left(\mathrm{km} \mathrm{s}^{-1}\right)\end{array} \\
\end{array}$ & $\begin{array}{c}\sigma_{\text {red }} \\
\left(\mathrm{km} \mathrm{s}^{-1}\right)\end{array}$ & $\begin{array}{c}\sigma_{\text {final }} \\
\left(\mathrm{km} \mathrm{s}^{-1}\right)\end{array}$ & Notes & \\
\hline IC 10 & $\ldots$ & $\ldots$ & $\ldots$ & $\ldots$ & $\ldots$ & 1,2 & $35.5 \pm 16.6$ \\
\hline IC 239 & $\ldots$ & $\ldots$ & $92.3 \pm 10.7$ & $\ldots$ & $\ldots$ & $3,6,12$ & $92.3 \pm 10.7$ \\
\hline IC 342 & $74.3 \pm 11.4$ & 1 & $\ldots$ & $\ldots$ & $\ldots$ & 2,7 & $74.3 \pm 11.4$ \\
\hline IC 356 & $141.0 \pm 27.3$ & 1 & $200.1 \pm 15.9$ & $142.0 \pm 9.2$ & $156.6 \pm 8.0$ & & $156.6 \pm 8.0$ \\
\hline IC 467 & $\cdots$ & $\ldots$ & $\cdots$ & $64.2 \pm 8.9$ & $64.2 \pm 8.9$ & 8 & $64.2 \pm 8.9$ \\
\hline IC 520 & $\cdots$ & $\cdots$ & $126.6 \pm 10.9$ & $146.5 \pm 9.3$ & $138.1 \pm 7.1$ & & $138.1 \pm 7.1$ \\
\hline IC 1727 & $\cdots$ & $\cdots$ & $\ldots$ & $136.8 \pm 9.2$ & $136.8 \pm 9.2$ & 6 & $136.8 \pm 9.2$ \\
\hline IC 2574 & $\ldots$ & $\ldots$ & $\ldots$ & $\ldots$ & $\ldots$ & 1,2 & $33.9 \pm 15.9$ \\
\hline NGC 63 & $\ldots$ & $\ldots$ & $\ldots$ & $25.6 \pm 8.8$ & $25.6 \pm 8.8$ & $2,7,12$ & $25.6 \pm 8.8$ \\
\hline NGC 147 & $22.0 \pm 5.0$ & 1 & $\ldots$ & $\ldots$ & $\ldots$ & 2,9 & $22.0 \pm 5.0$ \\
\hline NGC 185 & $19.9 \pm 2.4$ & 1 & $\ldots$ & $\ldots$ & $\ldots$ & 2 & $19.9 \pm 2.4$ \\
\hline NGC 205 & $23.3 \pm 3.7$ & 1 & $\ldots$ & $\ldots$ & $\ldots$ & 2,7 & $23.3 \pm 3.7$ \\
\hline NGC 221 & $72.1 \pm 1.9$ & 1 & $\ldots$ & $87.7 \pm 9.1$ & $87.7 \pm 9.1$ & 6 & $72.1 \pm 1.9$ \\
\hline NGC 224 & $169.8 \pm 5.2$ & 1 & $191.5 \pm 16.4$ & $186.7 \pm 9.9$ & $188.0 \pm 8.5$ & & $169.8 \pm 5.2$ \\
\hline NGC 266 & $\ldots$ & $\ldots$ & $209.3 \pm 16.3$ & $238.5 \pm 10.8$ & $229.6 \pm 9.0$ & & $229.6 \pm 9.0$ \\
\hline NGC 278 & $\ldots$ & $\ldots$ & $\ldots$ & $47.6 \pm 8.9$ & $47.6 \pm 8.9$ & 6 & $47.6 \pm 8.9$ \\
\hline NGC 315 & $266.3 \pm 22.8$ & 1 & $296.4 \pm 22.0$ & $306.0 \pm 12.2$ & $303.7 \pm 10.7$ & & $303.7 \pm 10.7$ \\
\hline NGC 404 & $40 \pm 3$ & 2 & $\ldots$ & $\ldots$ & $\ldots$ & 6,7 & $40 \pm 3$ \\
\hline NGC 410 & $299.7 \pm 7.4$ & 1 & $313.3 \pm 19.5$ & $287.1 \pm 11.7$ & $294.0 \pm 10.0$ & & $299.7 \pm 7.4$ \\
\hline NGC 428 & $\ldots$ & $\ldots$ & $\ldots$ & $26.3 \pm 8.5$ & $26.3 \pm 8.5$ & $2,7,12$ & $26.3 \pm 8.5$ \\
\hline NGC 474 & $163.4 \pm 5.4$ & 1 & $156.3 \pm 12.7$ & $160.4 \pm 9.5$ & $158.9 \pm 7.6$ & & $163.4 \pm 5.4$ \\
\hline NGC 488 & $199.2 \pm 9.8$ & 1 & $197.3 \pm 14.9$ & $185.8 \pm 9.9$ & $189.3 \pm 8.2$ & & $189.3 \pm 8.2$ \\
\hline NGC 507 & $307.7 \pm 9.7$ & 1 & $257.1 \pm 19.3$ & $288.9 \pm 11.8$ & $280.2 \pm 10.1$ & & $307.7 \pm 9.7$ \\
\hline NGC 514 & $\ldots$ & $\ldots$ & $\ldots$ & $54.6 \pm 8.6$ & $54.6 \pm 8.6$ & 6 & $54.6 \pm 8.6$ \\
\hline NGC 520 & $240 \pm 25$ & 1 & $\ldots$ & $40.6 \pm 8.9$ & $40.6 \pm 8.9$ & $2,4,7,12$ & $40.6 \pm 8.9$ \\
\hline NGC 521 & $\ldots$ & $\ldots$ & $214.9 \pm 16.0$ & $210.5 \pm 10.3$ & $211.8 \pm 8.7$ & & $211.8 \pm 8.7$ \\
\hline NGC 524 & $253.5 \pm 7.8$ & 1 & $250.0 \pm 19.1$ & $237.9 \pm 10.8$ & $240.8 \pm 9.4$ & & $253.5 \pm 7.8$ \\
\hline NGC 598 & $21 \pm 3$ & 3 & $\ldots$ & $20.0 \pm 8.5$ & $20.0 \pm 8.5$ & $2,7,11,12$ & $21 \pm 3$ \\
\hline NGC 628 & $72.2 \pm 7.8$ & 1 & $\ldots$ & $58.0 \pm 8.6$ & $58.0 \pm 8.6$ & 6,8 & $72.2 \pm 7.8$ \\
\hline NGC 660 & $128 \pm 6$ & 2 & $\ldots$ & $121.9 \pm 9.0$ & $121.9 \pm 9.0$ & $3,6,8$ & $128 \pm 6$ \\
\hline NGC 672 & $\ldots$ & $\ldots$ & $\ldots$ & $\ldots$ & $\ldots$ & 1,2 & $<64.3$ \\
\hline NGC 676 & $\ldots$ & $\ldots$ & $135.0 \pm 12.2$ & $156.5 \pm 10.3$ & $147.6 \pm 7.9$ & & $147.6 \pm 7.9$ \\
\hline NGC 697 & $\ldots$ & $\ldots$ & $\ldots$ & $75.0 \pm 8.9$ & $75.0 \pm 8.9$ & 6 & $75.0 \pm 8.9$ \\
\hline NGC 718 & $134 \pm 20$ & 1 & $\ldots$ & $117.4 \pm 9.5$ & $117.4 \pm 9.5$ & 6 & $117.4 \pm 9.5$ \\
\hline NGC 772 & $127.8 \pm 5.2$ & 1 & $\ldots$ & $145.1 \pm 9.3$ & $145.1 \pm 9.3$ & 6 & $127.8 \pm 5.2$ \\
\hline NGC 777 & $324.1 \pm 10.6$ & 1 & $308.5 \pm 22.8$ & $314.9 \pm 12.3$ & $313.5 \pm 10.8$ & & $324.1 \pm 10.6$ \\
\hline NGC 783 & $\ldots$ & $\ldots$ & $\ldots$ & $101.4 \pm 9.3$ & $101.4 \pm 9.3$ & 6 & $101.4 \pm 9.3$ \\
\hline NGC 784 & $\ldots$ & $\ldots$ & $\ldots$ & $\ldots$ & $\ldots$ & 1,2 & $35.5 \pm 16.6$ \\
\hline NGC 812 & $\ldots$ & $\ldots$ & $\ldots$ & $120.9 \pm 9.0$ & $120.9 \pm 9.0$ & 6 & $120.9 \pm 9.0$ \\
\hline NGC 818 & $\ldots$ & $\ldots$ & $133.7 \pm 12.3$ & $164.2 \pm 10.5$ & $151.3 \pm 8.0$ & & $151.3 \pm 8.0$ \\
\hline NGC 821 & $210.4 \pm 3.6$ & 1 & $210.1 \pm 14.1$ & $204.7 \pm 10.3$ & $206.6 \pm 8.3$ & & $210.4 \pm 3.6$ \\
\hline NGC 841 & $\ldots$ & $\ldots$ & $177.0 \pm 16.3$ & $153.4 \pm 9.3$ & $159.2 \pm 8.1$ & & $159.2 \pm 8.1$ \\
\hline NGC 864 & $65 \pm$ & 4 & $\ldots$ & $26.9 \pm 9.0$ & $26.9 \pm 9.0$ & $6,7,12$ & $26.9 \pm 9.0$ \\
\hline NGC 877 & $\ldots$ & $\ldots$ & $\ldots$ & $105.8 \pm 8.8$ & $105.8 \pm 8.8$ & 6 & $105.8 \pm 8.8$ \\
\hline NGC 890 & $229.2 \pm 9.9$ & 1 & $194.8 \pm 15.5$ & $218.5 \pm 10.6$ & $210.9 \pm 8.7$ & & $210.9 \pm 8.7$ \\
\hline NGC 891 & $73.1 \pm 10.2$ & 1 & $\ldots$ & $\ldots$ & $\ldots$ & 2 & $73.1 \pm 10.2$ \\
\hline NGC 925 & $\ldots$ & $\ldots$ & $\ldots$ & $\ldots$ & $\ldots$ & 1,2 & $<71.9$ \\
\hline NGC 959 & $\ldots$ & $\ldots$ & $\ldots$ & $\ldots$ & $\ldots$ & $1,2,7$ & $43.6 \pm 20.4$ \\
\hline NGC 972 & $\ldots$ & $\ldots$ & $\ldots$ & $102.8 \pm 9.6$ & $102.8 \pm 9.6$ & & $102.8 \pm 9.6$ \\
\hline NGC 1003 & $\ldots$ & $\ldots$ & $\ldots$ & $\ldots$ & $\ldots$ & 2,5 & $\ldots$ \\
\hline NGC 1023 & $204.5 \pm 4.2$ & 1 & $206.9 \pm 13.1$ & $224.5 \pm 10.5$ & $217.6 \pm 8.2$ & & $204.5 \pm 4.2$ \\
\hline NGC 1052 & $215 \pm 4$ & 2 & $229.7 \pm 14.9$ & $248.3 \pm 11.2$ & $241.6 \pm 9.0$ & & $215 \pm 4$ \\
\hline NGC 1055 & $80 \pm 15$ & 1 & $\ldots$ & $\ldots$ & $\ldots$ & 3,5 & $80 \pm 15$ \\
\hline NGC 1058 & $31 \pm 6$ & 2 & $\cdots$ & $62.2 \pm 8.4$ & $62.2 \pm 8.4$ & 6 & $31 \pm 6$ \\
\hline NGC 1068 & $198.7 \pm 17.0$ & 1 & $135.2 \pm 29.1$ & $165.2 \pm 9.6$ & $162.3 \pm 9.1$ & & $162.3 \pm 9.1$ \\
\hline NGC 1073 & $\ldots$ & $\ldots$ & $\ldots$ & $24.8 \pm 8.7$ & $24.8 \pm 8.7$ & $6,7,12$ & $24.8 \pm 8.7$ \\
\hline NGC 1156 & $\ldots$ & $\ldots$ & $\ldots$ & $\ldots$ & $\ldots$ & 1,2 & $35.9 \pm 16.8$ \\
\hline NGC 1161 & $286.7 \pm 16.4$ & 1 & $271.6 \pm 18.7$ & $253.8 \pm 11.1$ & $258.4 \pm 9.5$ & & $258.4 \pm 9.5$ \\
\hline NGC 1167 & $171 \pm$ & 5 & $210.4 \pm 15.0$ & $220.1 \pm 10.5$ & $216.9 \pm 8.6$ & & $216.9 \pm 8.6$ \\
\hline NGC 1169 & $152.5 \pm 25.4$ & 1 & $195.1 \pm 9.9$ & $168.1 \pm 9.7$ & $181.3 \pm 6.9$ & & $181.3 \pm 6.9$ \\
\hline NGC 1186 & $139.3 \pm 29.5$ & 1 & $\ldots$ & $119.8 \pm 9.5$ & $119.8 \pm 9.5$ & 6 & $119.8 \pm 9.5$ \\
\hline NGC 1275 & $258.9 \pm 13.4$ & 1 & $\ldots$ & $\ldots$ & $\ldots$ & 2 & $258.9 \pm 13.4$ \\
\hline NGC 1358 & $176.7 \pm 10.1$ & 1 & $244.3 \pm 16.8$ & $214.0 \pm 10.3$ & $222.3 \pm 8.8$ & & $222.3 \pm 8.8$ \\
\hline NGC 1560 & $\ldots$ & $\ldots$ & $\ldots$ & $\ldots$ & $\ldots$ & 1,2 & $33.9 \pm 15.9$ \\
\hline
\end{tabular}


Table 1

(Continued)

\begin{tabular}{|c|c|c|c|c|c|c|c|}
\hline \multirow[t]{2}{*}{ Galaxy } & \multicolumn{2}{|c|}{ Literature } & \multicolumn{4}{|c|}{ Palomar } & \multirow{2}{*}{$\begin{array}{c}\text { Adopted } \\
\sigma \\
\left(\mathrm{km} \mathrm{s}^{-1}\right)\end{array}$} \\
\hline & $\begin{array}{c}\sigma \\
\left(\mathrm{km} \mathrm{s}^{-1}\right)\end{array}$ & Reference & $\begin{array}{c}\begin{array}{c}\sigma_{\text {blue }} \\
\left(\mathrm{km} \mathrm{s}^{-1}\right)\end{array} \\
\end{array}$ & $\begin{array}{c}\sigma_{\text {red }} \\
\left(\mathrm{km} \mathrm{s}^{-1}\right)\end{array}$ & $\begin{array}{c}\sigma_{\text {final }} \\
\left(\mathrm{km} \mathrm{s}^{-1}\right)\end{array}$ & Notes & \\
\hline NGC 1569 & $\ldots$ & $\ldots$ & $\ldots$ & $\ldots$ & $\ldots$ & 1,2 & $44.0 \pm 20.6$ \\
\hline NGC 1667 & $187.2 \pm 27.0$ & 1 & $176.1 \pm 12.8$ & $164.2 \pm 10.3$ & $168.9 \pm 8.0$ & & $168.9 \pm 8.0$ \\
\hline NGC 1961 & $272.2 \pm 46.6$ & 1 & $196.1 \pm 15.3$ & $265.9 \pm 11.3$ & $241.3 \pm 9.1$ & & $241.3 \pm 9.1$ \\
\hline NGC 2146 & $121.3 \pm 33.9$ & 1 & $\ldots$ & $126.8 \pm 10.5$ & $126.8 \pm 10.5$ & 2 & $126.8 \pm 10.5$ \\
\hline NGC 2268 & & $\ldots$ & $112.3 \pm 15.9$ & $156.3 \pm 10.3$ & $143.3 \pm 8.6$ & & $143.3 \pm 8.6$ \\
\hline NGC 2273 & $122.7 \pm 9.9$ & 1 & $114.3 \pm 21.9$ & $156.7 \pm 10.4$ & $148.9 \pm 9.4$ & & $148.9 \pm 9.4$ \\
\hline NGC 2276 & $\ldots$ & $\ldots$ & $\ldots$ & $83.5 \pm 9.5$ & $83.5 \pm 9.5$ & 2 & $83.5 \pm 9.5$ \\
\hline NGC 2300 & $261.1 \pm 6.1$ & 1 & $263.0 \pm 19.1$ & $292.1 \pm 11.7$ & $284.2 \pm 10.0$ & & $261.1 \pm 6.1$ \\
\hline NGC 2336 & $141.5 \pm 12.1$ & 1 & $108.4 \pm 10.4$ & $122.0 \pm 9.0$ & $116.2 \pm 6.8$ & & $116.2 \pm 6.8$ \\
\hline NGC 2339 & $\ldots$ & $\ldots$ & $\ldots$ & $187.5 \pm 10.1$ & $187.5 \pm 10.1$ & 8 & $187.5 \pm 10.1$ \\
\hline NGC 2342 & $\ldots$ & $\ldots$ & $\ldots$ & $147.3 \pm 10.6$ & $147.3 \pm 10.6$ & 8 & $147.3 \pm 10.6$ \\
\hline NGC 2366 & $\ldots$ & $\ldots$ & $\ldots$ & $\ldots$ & $\ldots$ & 2 & $\ldots$ \\
\hline NGC 2403 & $\ldots$ & $\ldots$ & $\ldots$ & $\ldots$ & $\ldots$ & $1,6,7$ & $68.4 \pm 32.0$ \\
\hline NGC 2500 & $\ldots$ & $\ldots$ & $\ldots$ & $\ldots$ & $\ldots$ & $1,2,7$ & $47.1 \pm 22.1$ \\
\hline NGC 2537 & $\ldots$ & $\ldots$ & $\ldots$ & $63.0 \pm 9.1$ & $63.0 \pm 9.1$ & 2 & $63.0 \pm 9.1$ \\
\hline NGC 2541 & $53 \pm 10$ & 1 & $\ldots$ & $\ldots$ & $\ldots$ & 2 & $53 \pm 10$ \\
\hline NGC 2543 & $\ldots$ & $\ldots$ & $163.4 \pm 20.8$ & $101.3 \pm 9.7$ & $112.4 \pm 8.8$ & & $112.4 \pm 8.8$ \\
\hline NGC 2549 & $142.6 \pm 3.8$ & 1 & $154.0 \pm 11.5$ & $145.2 \pm 9.3$ & $148.7 \pm 7.2$ & & $142.6 \pm 3.8$ \\
\hline NGC 2634 & $181.1 \pm 4.7$ & 1 & $164.1 \pm 13.4$ & $188.8 \pm 10.0$ & $180.0 \pm 8.0$ & & $181.1 \pm 4.7$ \\
\hline NGC 2639 & $198.2 \pm 10.5$ & 1 & $183.9 \pm 13.0$ & $176.8 \pm 9.7$ & $179.3 \pm 7.8$ & & $179.3 \pm 7.8$ \\
\hline NGC 2655 & $162.5 \pm 11.2$ & 1 & $138.2 \pm 14.3$ & $169.8 \pm 9.7$ & $159.8 \pm 8.0$ & & $159.8 \pm 8.0$ \\
\hline NGC 2681 & $109.1 \pm 6.7$ & 1 & $132.8 \pm 13.4$ & $130.5 \pm 9.1$ & $131.2 \pm 7.5$ & & $109.1 \pm 6.7$ \\
\hline NGC 2683 & $116.4 \pm 10.9$ & 1 & $127.9 \pm 11.2$ & $131.7 \pm 9.0$ & $130.2 \pm 7.0$ & & $130.2 \pm 7.0$ \\
\hline NGC 2685 & $93.8 \pm 4.9$ & 1 & $123.5 \pm 10.0$ & $86.7 \pm 8.6$ & $102.3 \pm 6.5$ & & $93.8 \pm 4.9$ \\
\hline NGC 2715 & $\ldots$ & $\ldots$ & $\ldots$ & $84.6 \pm 9.0$ & $84.6 \pm 9.0$ & 6 & $84.6 \pm 9.0$ \\
\hline NGC 2742 & $65.6 \pm 28.9$ & 1 & $\ldots$ & $\ldots$ & $\ldots$ & 6,7 & $65.6 \pm 28.9$ \\
\hline NGC 2748 & $83 \pm 8$ & 6 & $\ldots$ & $96.4 \pm 9.5$ & $96.4 \pm 9.5$ & 6 & $83 \pm 8$ \\
\hline NGC 2750 & $\ldots$ & $\ldots$ & $\ldots$ & $52.4 \pm 9.5$ & $52.4 \pm 9.5$ & 8 & $52.4 \pm 9.5$ \\
\hline NGC 2768 & $181.8 \pm 3.6$ & 1 & $190.6 \pm 12.7$ & $196.0 \pm 10.1$ & $193.9 \pm 7.9$ & & $181.8 \pm 3.6$ \\
\hline NGC 2770 & $\ldots$ & $\ldots$ & $\ldots$ & $81.0 \pm 8.9$ & $81.0 \pm 8.9$ & 6 & $81.0 \pm 8.9$ \\
\hline NGC 2775 & $175.5 \pm 7.8$ & 1 & $167.1 \pm 12.3$ & $177.4 \pm 9.7$ & $173.5 \pm 7.6$ & & $173.5 \pm 7.6$ \\
\hline NGC 2776 & $75 \pm 11.3$ & 1 & $\ldots$ & $47.7 \pm 8.6$ & $47.7 \pm 8.6$ & 6 & $47.7 \pm 8.6$ \\
\hline NGC 2782 & $154.2 \pm 23.3$ & 1 & $122.5 \pm 30.6$ & $189.6 \pm 10.0$ & $183.1 \pm 9.5$ & & $183.1 \pm 9.5$ \\
\hline NGC 2787 & $202 \pm 5$ & 2 & $199.5 \pm 13.5$ & $197.6 \pm 10.1$ & $198.3 \pm 8.1$ & & $202 \pm 5$ \\
\hline NGC 2832 & $334.0 \pm 7.4$ & 1 & $331.9 \pm 21.6$ & $323.8 \pm 12.5$ & $325.8 \pm 10.8$ & & $334.0 \pm 7.4$ \\
\hline NGC 2841 & $222 \pm 4$ & 2 & $254.6 \pm 15.7$ & $222.8 \pm 10.5$ & $232.6 \pm 8.7$ & & $222 \pm 4$ \\
\hline NGC 2859 & $179.8 \pm 10.9$ & 1 & $180.1 \pm 13.1$ & $192.8 \pm 9.9$ & $188.2 \pm 7.9$ & & $188.2 \pm 7.9$ \\
\hline NGC 2903 & $89 \pm 4$ & 6 & $\ldots$ & $139.7 \pm 10.5$ & $139.7 \pm 10.5$ & 2 & $89 \pm 4$ \\
\hline NGC 2911 & $233.7 \pm 16.1$ & 1 & $265.8 \pm 16.1$ & $233.4 \pm 10.6$ & $243.2 \pm 8.9$ & & $243.2 \pm 8.9$ \\
\hline NGC 2950 & $185.4 \pm 9.8$ & 1 & $139.5 \pm 11.0$ & $182.1 \pm 9.9$ & $163.0 \pm 7.4$ & & $163.0 \pm 7.4$ \\
\hline NGC 2964 & $103 \pm 12$ & 6 & $\ldots$ & $109.4 \pm 9.8$ & $109.4 \pm 9.8$ & 6 & $109.4 \pm 9.8$ \\
\hline NGC 2967 & $181.9 \pm 24.6$ & 1 & $\ldots$ & $89.5 \pm 9.5$ & $89.5 \pm 9.5$ & 6 & $89.5 \pm 9.5$ \\
\hline NGC 2976 & $\ldots$ & $\ldots$ & $\ldots$ & $\ldots$ & $\ldots$ & $1,6,7$ & $36.0 \pm 16.8$ \\
\hline NGC 2977 & $\ldots$ & $\ldots$ & $\ldots$ & $104.5 \pm 9.3$ & $104.5 \pm 9.3$ & 6 & $104.5 \pm 9.3$ \\
\hline NGC 2985 & $140.8 \pm 4.7$ & 1 & $194.9 \pm 14.6$ & $190.6 \pm 10.0$ & $192.0 \pm 8.3$ & & $140.8 \pm 4.7$ \\
\hline NGC 3003 & $\ldots$ & $\ldots$ & $\ldots$ & $\ldots$ & $\ldots$ & $1,2,7$ & $44.1 \pm 20.6$ \\
\hline NGC 3027 & $\ldots$ & $\ldots$ & $\ldots$ & $25.6 \pm 9.1$ & $25.6 \pm 9.1$ & $1,6,7,12$ & $25.6 \pm 9.1$ \\
\hline NGC 3031 & $161.6 \pm 3.1$ & 1 & $159.1 \pm 11.0$ & $154.6 \pm 9.5$ & $156.5 \pm 7.2$ & & $161.6 \pm 3.1$ \\
\hline NGC 3034 & $129.5 \pm 27.8$ & 1 & $\ldots$ & $\ldots$ & $\ldots$ & 2 & $129.5 \pm 27.8$ \\
\hline NGC 3041 & $97 \pm 30$ & 1 & $\ldots$ & $105.4 \pm 9.4$ & $105.4 \pm 9.4$ & 6 & $105.4 \pm 9.4$ \\
\hline NGC 3043 & $\ldots$ & $\ldots$ & $\ldots$ & $51.9 \pm 9.0$ & $51.9 \pm 9.0$ & 2 & $51.9 \pm 9.0$ \\
\hline NGC 3073 & $34.8 \pm 3.9$ & 1 & $\ldots$ & $35.6 \pm 8.7$ & $35.6 \pm 8.7$ & $2,7,12$ & $35.6 \pm 8.7$ \\
\hline NGC 3077 & & $\ldots$ & $\ldots$ & $\ldots$ & $\ldots$ & $1,2,6,7$ & $32.4 \pm 15.2$ \\
\hline NGC 3079 & $145.7 \pm 9.7$ & 1 & $130.5 \pm 15.8$ & $206.1 \pm 10.7$ & $182.3 \pm 8.9$ & & $182.3 \pm 8.9$ \\
\hline NGC 3115 & $252.1 \pm 5.9$ & 1 & $258.6 \pm 17.1$ & $258.5 \pm 11.1$ & $258.5 \pm 9.3$ & & $252.1 \pm 5.9$ \\
\hline NGC 3147 & $261.3 \pm 22.2$ & 1 & $235.0 \pm 16.7$ & $214.1 \pm 10.2$ & $219.8 \pm 8.7$ & & $219.8 \pm 8.7$ \\
\hline NGC 3162 & $89 \pm 2$ & 6 & $141.5 \pm 26.2$ & $54.5 \pm 9.1$ & $63.9 \pm 8.6$ & & $89 \pm 2$ \\
\hline NGC 3166 & $112.3 \pm 23.1$ & 1 & $146.0 \pm 14.4$ & $156.6 \pm 9.5$ & $153.4 \pm 7.9$ & & $153.4 \pm 7.9$ \\
\hline NGC 3169 & $165.0 \pm 16.4$ & 1 & $177.4 \pm 17.7$ & $192.2 \pm 10.0$ & $188.6 \pm 8.7$ & & $188.6 \pm 8.7$ \\
\hline NGC 3184 & $\ldots$ & $\ldots$ & $\ldots$ & $43.3 \pm 8.9$ & $43.3 \pm 8.9$ & $2,7,12$ & $43.3 \pm 8.9$ \\
\hline NGC 3185 & $59.1 \pm 19.4$ & 1 & $\ldots$ & $79.3 \pm 9.2$ & $79.3 \pm 9.2$ & 6 & $79.3 \pm 9.2$ \\
\hline NGC 3190 & $169 \pm 11$ & 7 & $167.3 \pm 14.6$ & $198.0 \pm 10.1$ & $188.1 \pm 8.3$ & & $188.1 \pm 8.3$ \\
\hline NGC 3193 & $194.3 \pm 5.9$ & 1 & $171.3 \pm 12.5$ & $224.8 \pm 10.6$ & $202.4 \pm 8.1$ & & $194.3 \pm 5.9$ \\
\hline NGC 3198 & $65 \pm 10$ & 1 & $\ldots$ & $46.1 \pm 8.7$ & $46.1 \pm 8.7$ & $6,7,12$ & $46.1 \pm 8.7$ \\
\hline
\end{tabular}


Table 1

(Continued)

\begin{tabular}{|c|c|c|c|c|c|c|c|}
\hline \multirow[t]{2}{*}{ Galaxy } & \multicolumn{2}{|c|}{ Literature } & \multicolumn{4}{|c|}{ Palomar } & \multirow{2}{*}{$\begin{array}{c}\text { Adopted } \\
\sigma \\
\left(\mathrm{km} \mathrm{s}^{-1}\right)\end{array}$} \\
\hline & $\begin{array}{c}\sigma \\
\left(\mathrm{km} \mathrm{s}^{-1}\right) \\
\end{array}$ & Reference & $\begin{array}{c}\sigma_{\text {blue }} \\
\left(\mathrm{km} \mathrm{s}^{-1}\right)\end{array}$ & $\begin{array}{c}\sigma_{\text {red }} \\
\left(\mathrm{km} \mathrm{s}^{-1}\right) \\
\end{array}$ & $\begin{array}{c}\sigma_{\text {final }} \\
\left(\mathrm{km} \mathrm{s}^{-1}\right) \\
\end{array}$ & Notes & \\
\hline NGC 3226 & $193.5 \pm 5.1$ & 1 & $195.4 \pm 16.1$ & $236.9 \pm 10.6$ & $224.4 \pm 8.9$ & & $193.5 \pm 5.1$ \\
\hline NGC 3227 & $136 \pm 4$ & 8 & $\ldots$ & $\ldots$ & & 2 & $136 \pm 4$ \\
\hline NGC 3245 & $209.9 \pm 8.4$ & 1 & $209.3 \pm 17.1$ & $229.3 \pm 10.6$ & $223.7 \pm 9.0$ & & $209.9 \pm 8.4$ \\
\hline NGC 3254 & $117.8 \pm 4.1$ & 1 & $143.7 \pm 11.8$ & $141.5 \pm 9.2$ & $142.3 \pm 7.3$ & & $117.8 \pm 4.1$ \\
\hline NGC 3294 & $75.9 \pm 20.4$ & 1 & $\ldots$ & $56.4 \pm 8.7$ & $56.4 \pm 8.7$ & 6 & $56.4 \pm 8.7$ \\
\hline NGC 3301 & $121.1 \pm 9.8$ & 1 & $117.5 \pm 12.4$ & $140.4 \pm 9.2$ & $132.3 \pm 7.4$ & & $132.3 \pm 7.4$ \\
\hline NGC 3310 & $84 \pm 1$ & 6 & $\ldots$ & $96.6 \pm 10.1$ & $96.6 \pm 10.1$ & 6 & $84 \pm 1$ \\
\hline NGC 3319 & $\ldots$ & $\ldots$ & $\ldots$ & $87.4 \pm 9.2$ & $87.4 \pm 9.2$ & 8 & $87.4 \pm 9.2$ \\
\hline NGC 3338 & $86.9 \pm 17.2$ & 1 & $\ldots$ & $120.6 \pm 9.6$ & $120.6 \pm 9.6$ & 6 & $120.6 \pm 9.6$ \\
\hline NGC 3344 & $\cdots$ & $\ldots$ & $\ldots$ & $73.6 \pm 9.1$ & $73.6 \pm 9.1$ & 6 & $73.6 \pm 9.1$ \\
\hline NGC 3346 & $48 \pm$ & 4 & $\cdots$ & $76.3 \pm 8.4$ & $76.3 \pm 8.4$ & 8 & $76.3 \pm 8.4$ \\
\hline NGC 3348 & $236.4 \pm 10.4$ & 1 & $\ldots$ & $193.7 \pm 12.4$ & $193.7 \pm 12.4$ & 3,8 & $236.4 \pm 10.4$ \\
\hline NGC 3351 & $98.5 \pm 20.3$ & 1 & $\ldots$ & $119.9 \pm 9.0$ & $119.9 \pm 9.0$ & 6 & $119.9 \pm 9.0$ \\
\hline NGC 3359 & $\ldots$ & $\ldots$ & $\cdots$ & $55.3 \pm 8.9$ & $55.3 \pm 8.9$ & 8 & $55.3 \pm 8.9$ \\
\hline NGC 3367 & $\cdots$ & $\cdots$ & $\cdots$ & $61.2 \pm 10.1$ & $61.2 \pm 10.1$ & 2 & $61.2 \pm 10.1$ \\
\hline NGC 3368 & $128.2 \pm 4.0$ & 1 & $\cdots$ & $126.3 \pm 9.0$ & $126.3 \pm 9.0$ & 6 & $128.2 \pm 4.0$ \\
\hline NGC 3370 & $48.0 \pm 27.9$ & 1 & $\cdots$ & $94.6 \pm 9.4$ & $94.6 \pm 9.4$ & 6 & $94.6 \pm 9.4$ \\
\hline NGC 3377 & $138.7 \pm 2.6$ & 1 & $131.0 \pm 12.3$ & $173.0 \pm 9.7$ & $156.9 \pm 7.6$ & & $138.7 \pm 2.6$ \\
\hline NGC 3379 & $206.7 \pm 2.4$ & 1 & $247.2 \pm 16.0$ & $191.7 \pm 9.9$ & $207.1 \pm 8.4$ & & $206.7 \pm 2.4$ \\
\hline NGC 3384 & $148.4 \pm 3.4$ & 1 & $161.2 \pm 11.5$ & $136.7 \pm 9.2$ & $146.3 \pm 7.2$ & & $148.4 \pm 3.4$ \\
\hline NGC 3389 & $\ldots$ & $\cdots$ & $69.3 \pm 14.6$ & $\ldots$ & $69.3 \pm 14.6$ & $3,6,9,12$ & $69.3 \pm 14.6$ \\
\hline NGC 3395 & $\cdots$ & $\ldots$ & $96.5 \pm 14.6$ & $\cdots$ & $96.5 \pm 14.6$ & $3,6,9,12$ & $96.5 \pm 14.6$ \\
\hline NGC 3412 & $100.7 \pm 2.3$ & 1 & $\ldots$ & $108.1 \pm 8.8$ & $108.1 \pm 8.8$ & 6 & $100.7 \pm 2.3$ \\
\hline NGC 3414 & $236.8 \pm 7.5$ & 1 & $272.0 \pm 17.9$ & $256.2 \pm 11.1$ & $260.6 \pm 9.4$ & & $236.8 \pm 7.5$ \\
\hline NGC 3423 & $49 \pm$ & 4 & $\ldots$ & $54.6 \pm 8.5$ & $54.6 \pm 8.5$ & 6 & $54.6 \pm 8.5$ \\
\hline NGC 3430 & $\ldots$ & $\ldots$ & $\cdots$ & $50.4 \pm 8.9$ & $50.4 \pm 8.9$ & 6 & $50.4 \pm 8.9$ \\
\hline NGC 3432 & $\ldots$ & $\cdots$ & $\cdots$ & $\cdots$ & $\cdots$ & 1,2 & $37.0 \pm 17.3$ \\
\hline NGC 3433 & $\ldots$ & $\ldots$ & $\cdots$ & $80.0 \pm 8.7$ & $80.0 \pm 8.7$ & 6 & $80.0 \pm 8.7$ \\
\hline NGC 3448 & $\ldots$ & $\ldots$ & $\ldots$ & $\ldots$ & $\ldots$ & 1,2 & $50.7 \pm 23.8$ \\
\hline NGC 3486 & $65 \pm 3$ & 2 & $\ldots$ & $68.2 \pm 8.4$ & $68.2 \pm 8.4$ & 6 & $65 \pm 3$ \\
\hline NGC 3489 & $112 \pm 3$ & 2 & $\ldots$ & $120.9 \pm 9.0$ & $120.9 \pm 9.0$ & 6 & $112 \pm 3$ \\
\hline NGC 3495 & $\ldots$ & $\ldots$ & $\ldots$ & $60.4 \pm 9.0$ & $60.4 \pm 9.0$ & 6 & $60.4 \pm 9.0$ \\
\hline NGC 3504 & $123.9 \pm 18.2$ & 1 & $\cdots$ & $119.3 \pm 10.3$ & $119.3 \pm 10.3$ & 6 & $119.3 \pm 10.3$ \\
\hline NGC 3507 & $\ldots$ & $\ldots$ & $\ldots$ & $85.5 \pm 9.0$ & $85.5 \pm 9.0$ & 6 & $85.5 \pm 9.0$ \\
\hline NGC 3516 & $181 \pm 5$ & 8 & $147.6 \pm 21.6$ & $\ldots$ & $147.6 \pm 21.6$ & 2 & $181 \pm 5$ \\
\hline NGC 3521 & $180 \pm 39$ & 9 & $131.2 \pm 11.4$ & $130.1 \pm 9.1$ & $130.5 \pm 7.1$ & & $130.5 \pm 7.1$ \\
\hline NGC 3556 & $\ldots$ & $\ldots$ & $\cdots$ & $79.4 \pm 9.6$ & $79.4 \pm 9.6$ & 2 & $79.4 \pm 9.6$ \\
\hline NGC 3583 & $142.9 \pm 47.3$ & 1 & $\cdots$ & $131.7 \pm 10.1$ & $131.7 \pm 10.1$ & 6 & $131.7 \pm 10.1$ \\
\hline NGC 3593 & $54.4 \pm 7.2$ & 1 & $\cdots$ & $106.0 \pm 8.9$ & $106.0 \pm 8.9$ & 6 & $54.4 \pm 7.2$ \\
\hline NGC 3596 & $\ldots$ & $\ldots$ & $\cdots$ & $103.0 \pm 9.4$ & $103.0 \pm 9.4$ & 6 & $103.0 \pm 9.4$ \\
\hline NGC 3600 & $\ldots$ & $\ldots$ & $\ldots$ & $49.8 \pm 9.1$ & $49.8 \pm 9.1$ & 8 & $49.8 \pm 9.1$ \\
\hline NGC 3607 & $224.9 \pm 8.8$ & 1 & $227.6 \pm 15.4$ & $234.5 \pm 10.7$ & $232.3 \pm 8.8$ & & $232.3 \pm 8.8$ \\
\hline NGC 3608 & $192.2 \pm 3.5$ & 1 & $195.4 \pm 13.7$ & $241.9 \pm 10.8$ & $224.1 \pm 8.5$ & & $192.2 \pm 3.5$ \\
\hline NGC 3610 & $161.2 \pm 4.6$ & 1 & $159.6 \pm 13.1$ & $170.2 \pm 10.7$ & $166.0 \pm 8.3$ & & $161.2 \pm 4.6$ \\
\hline NGC 3613 & $210.3 \pm 9.0$ & 1 & $218.4 \pm 16.2$ & $220.8 \pm 10.5$ & $220.1 \pm 8.8$ & & $220.1 \pm 8.8$ \\
\hline NGC 3623 & $138 \pm 3$ & 2 & $177.5 \pm 11.6$ & $137.7 \pm 9.1$ & $152.9 \pm 7.2$ & & $138 \pm 3$ \\
\hline NGC 3626 & $164.2 \pm 11.1$ & 1 & $112.2 \pm 14.4$ & $161.2 \pm 9.6$ & $146.1 \pm 8.0$ & & $146.1 \pm 8.0$ \\
\hline NGC 3627 & $124 \pm 3$ & 2 & $137.9 \pm 15.4$ & $154.5 \pm 9.7$ & $149.8 \pm 8.2$ & & $124 \pm 3$ \\
\hline NGC 3628 & $170.6 \pm 70.6$ & 1 & $\ldots$ & $77.3 \pm 8.9$ & $77.3 \pm 8.9$ & 6 & $77.3 \pm 8.9$ \\
\hline NGC 3631 & $\ldots$ & $\ldots$ & $\ldots$ & $43.9 \pm 8.6$ & $43.9 \pm 8.6$ & $6,7,12$ & $43.9 \pm 8.6$ \\
\hline NGC 3640 & $181.6 \pm 4.5$ & 1 & $189.3 \pm 14.1$ & $221.1 \pm 10.7$ & $209.5 \pm 8.5$ & & $181.6 \pm 4.5$ \\
\hline NGC 3642 & $158.1 \pm 32.7$ & 1 & $124.2 \pm 14.5$ & $69.5 \pm 9.1$ & $85.0 \pm 7.7$ & & $85.0 \pm 7.7$ \\
\hline NGC 3646 & $\ldots$ & $\ldots$ & $137.1 \pm 11.5$ & $164.3 \pm 9.6$ & $153.1 \pm 7.4$ & & $153.1 \pm 7.4$ \\
\hline NGC 3652 & $\ldots$ & $\ldots$ & $\ldots$ & $56.4 \pm 8.3$ & $56.4 \pm 8.3$ & 8 & $56.4 \pm 8.3$ \\
\hline NGC 3655 & $\ldots$ & $\ldots$ & $\ldots$ & $91.1 \pm 9.4$ & $91.1 \pm 9.4$ & 6 & $91.1 \pm 9.4$ \\
\hline NGC 3665 & $184.1 \pm 9.2$ & 1 & $215.5 \pm 17.3$ & $245.2 \pm 10.9$ & $236.8 \pm 9.2$ & & $236.8 \pm 9.2$ \\
\hline NGC 3666 & $\ldots$ & $\cdots$ & $\cdots$ & $60.6 \pm 8.7$ & $60.6 \pm 8.7$ & 6 & $60.6 \pm 8.7$ \\
\hline NGC 3675 & $108 \pm 4$ & 2 & $\ldots$ & $102.5 \pm 8.7$ & $102.5 \pm 8.7$ & 6 & $108 \pm 4$ \\
\hline NGC 3681 & $\ldots$ & $\ldots$ & $\ldots$ & $92.0 \pm 8.9$ & $92.0 \pm 8.9$ & 6 & $92.0 \pm 8.9$ \\
\hline NGC 3684 & $\ldots$ & $\ldots$ & $\ldots$ & $43.0 \pm 8.9$ & $43.0 \pm 8.9$ & $6,7,12$ & $43.0 \pm 8.9$ \\
\hline NGC 3686 & $\ldots$ & $\cdots$ & $\ldots$ & $151.3 \pm 9.7$ & $151.3 \pm 9.7$ & 2 & $151.3 \pm 9.7$ \\
\hline NGC 3690 & $\ldots$ & $\ldots$ & $\ldots$ & $143.9 \pm 11.3$ & $143.9 \pm 11.3$ & 2 & $143.9 \pm 11.3$ \\
\hline NGC 3692 & $\ldots$ & $\cdots$ & $\cdots$ & $113.6 \pm 9.5$ & $113.6 \pm 9.5$ & 6 & $113.6 \pm 9.5$ \\
\hline NGC 3705 & $109 \pm 9$ & 1 & $\ldots$ & $116.0 \pm 9.0$ & $116.0 \pm 9.0$ & 6 & $116.0 \pm 9.0$ \\
\hline
\end{tabular}


Table 1

(Continued)

\begin{tabular}{|c|c|c|c|c|c|c|c|}
\hline \multirow[t]{2}{*}{ Galaxy } & \multicolumn{2}{|c|}{ Literature } & \multicolumn{4}{|c|}{ Palomar } & \multirow{2}{*}{$\begin{array}{c}\text { Adopted } \\
\sigma \\
\left(\mathrm{km} \mathrm{s}^{-1}\right)\end{array}$} \\
\hline & $\begin{array}{c}\sigma \\
\left(\mathrm{km} \mathrm{s}^{-1}\right)\end{array}$ & Reference & $\begin{array}{c}\begin{array}{c}\sigma_{\text {blue }} \\
\left(\mathrm{km} \mathrm{s}^{-1}\right)\end{array} \\
\end{array}$ & $\begin{array}{c}\sigma_{\text {red }} \\
\left(\mathrm{km} \mathrm{s}^{-1}\right)\end{array}$ & $\begin{array}{c}\sigma_{\text {final }} \\
\left(\mathrm{km} \mathrm{s}^{-1}\right)\end{array}$ & Notes & \\
\hline NGC 3718 & $169.9 \pm 18.1$ & 1 & $\ldots$ & $158.1 \pm 9.6$ & $158.1 \pm 9.6$ & 2 & $158.1 \pm 9.6$ \\
\hline NGC 3726 & $69.5 \pm 24.8$ & 1 & $\ldots$ & $41.5 \pm 9.2$ & $41.5 \pm 9.2$ & $6,7,12$ & $41.5 \pm 9.2$ \\
\hline NGC 3729 & $\ldots$ & $\ldots$ & $\ldots$ & $76.2 \pm 9.1$ & $76.2 \pm 9.1$ & 6 & $76.2 \pm 9.1$ \\
\hline NGC 3735 & $\ldots$ & $\ldots$ & $124.4 \pm 15.0$ & $148.0 \pm 10.1$ & $140.6 \pm 8.4$ & & $140.6 \pm 8.4$ \\
\hline NGC 3738 & $\ldots$ & $\ldots$ & $\ldots$ & $49.1 \pm 9.1$ & $49.1 \pm 9.1$ & 2 & $49.1 \pm 9.1$ \\
\hline NGC 3756 & $\ldots$ & $\ldots$ & $\ldots$ & $47.6 \pm 8.5$ & $47.6 \pm 8.5$ & 6 & $47.6 \pm 8.5$ \\
\hline NGC 3780 & $\ldots$ & $\ldots$ & $\ldots$ & $89.8 \pm 9.4$ & $89.8 \pm 9.4$ & 6 & $89.8 \pm 9.4$ \\
\hline NGC 3810 & $64.6 \pm 8.7$ & 1 & $\ldots$ & $93.8 \pm 9.5$ & $93.8 \pm 9.5$ & 6 & $64.6 \pm 8.7$ \\
\hline NGC 3813 & $\ldots$ & $\ldots$ & $\ldots$ & $72.1 \pm 9.2$ & $72.1 \pm 9.2$ & 6 & $72.1 \pm 9.2$ \\
\hline NGC 3838 & $144.3 \pm 12.8$ & 1 & $116.0 \pm 10.7$ & $161.4 \pm 9.5$ & $141.4 \pm 7.1$ & & $141.4 \pm 7.1$ \\
\hline NGC 3877 & $\ldots$ & $\ldots$ & $\ldots$ & $86.1 \pm 9.2$ & $86.1 \pm 9.2$ & 6 & $86.1 \pm 9.2$ \\
\hline NGC 3884 & $\ldots$ & $\ldots$ & $193.0 \pm 13.7$ & $217.3 \pm 10.5$ & $208.3 \pm 8.3$ & & $208.3 \pm 8.3$ \\
\hline NGC 3893 & $105.7 \pm 23.8$ & 1 & $\ldots$ & $85.3 \pm 8.7$ & $85.3 \pm 8.7$ & 6 & $85.3 \pm 8.7$ \\
\hline NGC 3898 & $206.5 \pm 7.4$ & 1 & $208.7 \pm 14.1$ & $189.6 \pm 9.9$ & $195.9 \pm 8.1$ & & $206.5 \pm 7.4$ \\
\hline NGC 3900 & $\ldots$ & $\ldots$ & $127.3 \pm 11.6$ & $146.8 \pm 9.3$ & $139.2 \pm 7.3$ & & $139.2 \pm 7.3$ \\
\hline NGC 3917 & $\ldots$ & $\ldots$ & $\ldots$ & $38.0 \pm 8.5$ & $38.0 \pm 8.5$ & $6,7,12$ & $38.0 \pm 8.5$ \\
\hline NGC 3938 & $29.1 \pm 4.9$ & 1 & $\ldots$ & $62.8 \pm 8.4$ & $62.8 \pm 8.4$ & 6 & $29.1 \pm 4.9$ \\
\hline NGC 3941 & $168.7 \pm 13.1$ & 1 & $148.6 \pm 11.0$ & $122.6 \pm 9.0$ & $133.0 \pm 7.0$ & & $133.0 \pm 7.0$ \\
\hline NGC 3945 & $174.4 \pm 10.2$ & 1 & $187.2 \pm 12.4$ & $194.3 \pm 10.0$ & $191.5 \pm 7.8$ & & $191.5 \pm 7.8$ \\
\hline NGC 3949 & $82 \pm 2$ & 6 & $\ldots$ & $55.6 \pm 8.7$ & $55.6 \pm 8.7$ & 6 & $82 \pm 2$ \\
\hline NGC 3953 & $116 \pm 3$ & 6 & $\ldots$ & $129.2 \pm 9.0$ & $129.2 \pm 9.0$ & 6 & $116 \pm 3$ \\
\hline NGC 3963 & $\ldots$ & $\ldots$ & $\ldots$ & $40.9 \pm 8.7$ & $40.9 \pm 8.7$ & $6,7,12$ & $40.9 \pm 8.7$ \\
\hline NGC 3976 & $\ldots$ & $\ldots$ & $221.4 \pm 18.2$ & $180.0 \pm 9.9$ & $189.5 \pm 8.7$ & & $189.5 \pm 8.7$ \\
\hline NGC 3982 & $73 \pm 4$ & 2 & $\ldots$ & $87.3 \pm 9.0$ & $87.3 \pm 9.0$ & 6 & $73 \pm 4$ \\
\hline NGC 3992 & $140 \pm 20$ & 10 & $186.3 \pm 11.4$ & $124.2 \pm 9.1$ & $148.4 \pm 7.1$ & & $148.4 \pm 7.1$ \\
\hline NGC 3998 & $304.6 \pm 10$ & 1 & $311.1 \pm 22.1$ & $\ldots$ & $311.1 \pm 22.1$ & 9 & $304.6 \pm 10$ \\
\hline NGC 4013 & $\ldots$ & $\ldots$ & $\ldots$ & $86.5 \pm 8.6$ & $86.5 \pm 8.6$ & 6 & $86.5 \pm 8.6$ \\
\hline NGC 4026 & $177.2 \pm 4.5$ & 1 & $201.4 \pm 15.7$ & $169.2 \pm 9.7$ & $178.1 \pm 8.3$ & & $177.2 \pm 4.5$ \\
\hline NGC 4036 & $181.1 \pm 8.3$ & 1 & $215.4 \pm 13.1$ & $214.9 \pm 10.6$ & $215.1 \pm 8.2$ & & $215.1 \pm 8.2$ \\
\hline NGC 4041 & $95 \pm 5$ & 11 & $\ldots$ & $80.1 \pm 9.0$ & $80.1 \pm 9.0$ & 6,10 & $95 \pm 5$ \\
\hline NGC 4051 & $89 \pm 3$ & 8 & $\ldots$ & $127.0 \pm 10.1$ & $127.0 \pm 10.1$ & 2 & $89 \pm 3$ \\
\hline NGC 4062 & $93.2 \pm 7.7$ & 1 & $\ldots$ & $51.9 \pm 8.4$ & $51.9 \pm 8.4$ & 6 & $93.2 \pm 7.7$ \\
\hline NGC 4064 & $\ldots$ & $\ldots$ & $\ldots$ & $78.2 \pm 8.9$ & $78.2 \pm 8.9$ & 2 & $78.2 \pm 8.9$ \\
\hline NGC 4088 & $77 \pm 2$ & 6 & $\ldots$ & $54.3 \pm 8.9$ & $54.3 \pm 8.9$ & 6 & $77 \pm 2$ \\
\hline NGC 4096 & $\ldots$ & $\ldots$ & $\ldots$ & $79.5 \pm 8.7$ & $79.5 \pm 8.7$ & 6 & $79.5 \pm 8.7$ \\
\hline NGC 4100 & $\ldots$ & $\ldots$ & $\ldots$ & $75.5 \pm 9.4$ & $75.5 \pm 9.4$ & 8 & $75.5 \pm 9.4$ \\
\hline NGC 4102 & $150 \pm$ & 4 & $162.9 \pm 24.5$ & $176.4 \pm 10.4$ & $174.3 \pm 9.6$ & & $174.3 \pm 9.6$ \\
\hline NGC 4111 & $147.9 \pm 4.0$ & 1 & $130.6 \pm 11.4$ & $147.1 \pm 9.3$ & $140.5 \pm 7.2$ & & $147.9 \pm 4.0$ \\
\hline NGC 4123 & $\ldots$ & $\ldots$ & $\ldots$ & $25.6 \pm 10.1$ & $25.6 \pm 10.1$ & $6,7,8,12$ & $25.6 \pm 10.1$ \\
\hline NGC 4124 & $68.7 \pm 13.5$ & 1 & $\ldots$ & $50.2 \pm 8.5$ & $50.2 \pm 8.5$ & 6 & $50.2 \pm 8.5$ \\
\hline NGC 4125 & $226.7 \pm 7.6$ & 1 & $235.7 \pm 15.4$ & $273.7 \pm 11.8$ & $259.6 \pm 9.4$ & & $226.7 \pm 7.6$ \\
\hline NGC 4136 & $\ldots$ & $\ldots$ & $\ldots$ & $38.4 \pm 8.7$ & $38.4 \pm 8.7$ & $6,7,8,12$ & $38.4 \pm 8.7$ \\
\hline NGC 4138 & $140.1 \pm 15.8$ & 1 & $130.2 \pm 11.5$ & $115.3 \pm 8.9$ & $120.9 \pm 7.0$ & & $120.9 \pm 7.0$ \\
\hline NGC 4143 & $214.4 \pm 15.5$ & 1 & $232.8 \pm 14.7$ & $193.0 \pm 9.6$ & $204.9 \pm 8.0$ & & $204.9 \pm 8.0$ \\
\hline NGC 4144 & $\ldots$ & $\ldots$ & $\ldots$ & $\ldots$ & $\ldots$ & 1,2 & $<64.3$ \\
\hline NGC 4145 & $\ldots$ & $\ldots$ & $\ldots$ & $\ldots$ & $\ldots$ & 2,3 & $\ldots$ \\
\hline NGC 4150 & $87 \pm 3$ & 2 & $\ldots$ & $86.2 \pm 9.4$ & $86.2 \pm 9.4$ & 6 & $87 \pm 3$ \\
\hline NGC 4151 & $97 \pm 3$ & 8 & $\ldots$ & $\ldots$ & $\ldots$ & 2 & $97 \pm 3$ \\
\hline NGC 4152 & $\ldots$ & $\ldots$ & $\ldots$ & $62.3 \pm 9.0$ & $62.3 \pm 9.0$ & 6 & $62.3 \pm 9.0$ \\
\hline NGC 4157 & $90.1 \pm 4.4$ & 1 & $\ldots$ & $106.1 \pm 8.8$ & $106.1 \pm 8.8$ & 6 & $90.1 \pm 4.4$ \\
\hline NGC 4162 & $\ldots$ & $\ldots$ & $\ldots$ & $76.1 \pm 8.9$ & $76.1 \pm 8.9$ & 6 & $76.1 \pm 8.9$ \\
\hline NGC 4168 & $183.9 \pm 3.7$ & 1 & $169.4 \pm 13.3$ & $199.6 \pm 10.2$ & $188.4 \pm 8.1$ & & $183.9 \pm 3.7$ \\
\hline NGC 4169 & $215.9 \pm 30.6$ & 1 & $187.6 \pm 14.4$ & $180.8 \pm 9.9$ & $183.0 \pm 8.2$ & & $183.0 \pm 8.2$ \\
\hline NGC 4178 & $\ldots$ & $\ldots$ & $\ldots$ & $25.7 \pm 9.1$ & $25.7 \pm 9.1$ & $2,7,12$ & $25.7 \pm 9.1$ \\
\hline NGC 4179 & $157.3 \pm 7.9$ & 1 & $167.4 \pm 12.3$ & $179.9 \pm 9.8$ & $175.0 \pm 7.7$ & & $175.0 \pm 7.7$ \\
\hline NGC 4183 & $\ldots$ & $\ldots$ & $\ldots$ & $\ldots$ & $\ldots$ & $1,2,7$ & $34.4 \pm 16.1$ \\
\hline NGC 4192 & $132.4 \pm 7.1$ & 1 & $159.7 \pm 11.6$ & $115.6 \pm 8.9$ & $131.9 \pm 7.1$ & & $131.9 \pm 7.1$ \\
\hline NGC 4203 & $167 \pm 3$ & 2 & $157.7 \pm 11.6$ & $201.4 \pm 10.1$ & $182.6 \pm 7.6$ & & $167 \pm 3$ \\
\hline NGC 4212 & $75 \pm 2$ & 6 & $\ldots$ & $60.9 \pm 9.1$ & $60.9 \pm 9.1$ & 6 & $75 \pm 2$ \\
\hline NGC 4214 & $\ldots$ & $\ldots$ & $\ldots$ & $\ldots$ & $\ldots$ & 1,2 & $51.6 \pm 24.2$ \\
\hline NGC 4216 & $206.8 \pm 9.6$ & 1 & $208.0 \pm 13.0$ & $190.9 \pm 9.9$ & $197.2 \pm 7.9$ & & $197.2 \pm 7.9$ \\
\hline NGC 4217 & $91.3 \pm 4.4$ & 1 & $\ldots$ & $121.1 \pm 9.4$ & $121.1 \pm 9.4$ & 6 & $91.3 \pm 4.4$ \\
\hline NGC 4220 & $124.7 \pm 14.7$ & 1 & $133.3 \pm 11.7$ & $90.1 \pm 8.7$ & $105.5 \pm 7.0$ & & $105.5 \pm 7.0$ \\
\hline NGC 4235 & $151 \pm 10$ & 12 & $134.1 \pm 12.1$ & $\ldots$ & $134.1 \pm 12.1$ & 9 & $151 \pm 10$ \\
\hline
\end{tabular}


Table 1

(Continued)

\begin{tabular}{|c|c|c|c|c|c|c|c|}
\hline \multirow[t]{2}{*}{ Galaxy } & \multicolumn{2}{|c|}{ Literature } & \multicolumn{4}{|c|}{ Palomar } & \multirow{2}{*}{$\begin{array}{c}\text { Adopted } \\
\sigma \\
\left(\mathrm{km} \mathrm{s}^{-1}\right)\end{array}$} \\
\hline & $\begin{array}{c}\sigma \\
\left(\mathrm{km} \mathrm{s}^{-1}\right) \\
\end{array}$ & Reference & $\begin{array}{c}\sigma_{\text {blue }} \\
\left(\mathrm{km} \mathrm{s}^{-1}\right)\end{array}$ & $\begin{array}{c}\sigma_{\text {red }} \\
\left(\mathrm{km} \mathrm{s}^{-1}\right)\end{array}$ & $\begin{array}{c}\sigma_{\text {final }} \\
\left(\mathrm{km} \mathrm{s}^{-1}\right)\end{array}$ & Notes & \\
\hline NGC 4236 & $\ldots$ & $\ldots$ & $\ldots$ & $\ldots$ & $\cdots$ & 1,2 & $<62.8$ \\
\hline NGC 4242 & $\cdots$ & $\ldots$ & $\ldots$ & $\ldots$ & $\ldots$ & 2 & $\ldots$ \\
\hline NGC 4244 & $\ldots$ & $\ldots$ & $\ldots$ & $36.8 \pm 9.2$ & $36.8 \pm 9.2$ & $6,7,12$ & $36.8 \pm 9.2$ \\
\hline NGC 4245 & $\ldots$ & $\ldots$ & $\ldots$ & $82.7 \pm 8.6$ & $82.7 \pm 8.6$ & 6 & $82.7 \pm 8.6$ \\
\hline NGC 4251 & $119.4 \pm 5.1$ & 1 & $122.2 \pm 11.1$ & $137.2 \pm 9.1$ & $131.2 \pm 7.0$ & & $119.4 \pm 5.1$ \\
\hline NGC 4254 & $130.1 \pm 15.3$ & 1 & $\cdots$ & $83.4 \pm 9.2$ & $83.4 \pm 9.2$ & 6 & $83.4 \pm 9.2$ \\
\hline NGC 4258 & $148 \pm 4$ & 6 & $151.3 \pm 13.0$ & $122.7 \pm 9.7$ & $132.9 \pm 7.8$ & & $148 \pm 4$ \\
\hline NGC 4261 & $308.8 \pm 5.8$ & 1 & $311.6 \pm 22.7$ & $301.0 \pm 12.0$ & $303.3 \pm 10.6$ & & $308.8 \pm 5.8$ \\
\hline NGC 4262 & $189.7 \pm 14.3$ & 1 & $197.6 \pm 15.6$ & $229.8 \pm 10.7$ & $219.5 \pm 8.8$ & & $219.5 \pm 8.8$ \\
\hline NGC 4267 & $164.6 \pm 6.1$ & 1 & $168.4 \pm 12.3$ & $153.4 \pm 9.4$ & $158.9 \pm 7.5$ & & $164.6 \pm 6.1$ \\
\hline NGC 4273 & $\ldots$ & $\ldots$ & $\ldots$ & $58.1 \pm 9.5$ & $58.1 \pm 9.5$ & 2 & $58.1 \pm 9.5$ \\
\hline NGC 4274 & $137.2 \pm 11.9$ & 1 & $147.3 \pm 12.0$ & $71.1 \pm 8.5$ & $96.6 \pm 6.9$ & & $96.6 \pm 6.9$ \\
\hline NGC 4278 & $261 \pm 8$ & 2 & $258.4 \pm 19.5$ & $253.5 \pm 11.0$ & $254.7 \pm 9.6$ & & $261 \pm 8$ \\
\hline NGC 4281 & $280.5 \pm 14.2$ & 1 & $270.1 \pm 20.9$ & $245.3 \pm 11.0$ & $250.7 \pm 9.7$ & & $250.7 \pm 9.7$ \\
\hline NGC 4291 & $285.3 \pm 5.7$ & 1 & $285.6 \pm 20.7$ & $326.7 \pm 13.1$ & $314.9 \pm 11.1$ & & $285.3 \pm 5.7$ \\
\hline NGC 4293 & $148.6 \pm 25.9$ & 1 & $\cdots$ & $112.2 \pm 9.2$ & $112.2 \pm 9.2$ & 6 & $112.2 \pm 9.2$ \\
\hline NGC 4298 & $\ldots$ & $\cdots$ & $\ldots$ & $42.2 \pm 8.7$ & $42.2 \pm 8.7$ & $6,7,12$ & $42.2 \pm 8.7$ \\
\hline NGC 4303 & $84 \pm 3$ & 6 & $\cdots$ & $79.5 \pm 8.5$ & $79.5 \pm 8.5$ & 6 & $84 \pm 3$ \\
\hline NGC 4314 & $117 \pm 4$ & 2 & $118.8 \pm 10.9$ & $105.5 \pm 8.5$ & $110.5 \pm 6.7$ & & $117 \pm 4$ \\
\hline NGC 4321 & $83 \pm 3$ & 6 & $\ldots$ & $112.7 \pm 8.9$ & $112.7 \pm 8.9$ & 6 & $83 \pm 3$ \\
\hline NGC 4324 & $98.0 \pm 3.5$ & 1 & $\ldots$ & $74.2 \pm 8.9$ & $74.2 \pm 8.9$ & 6 & $98.0 \pm 3.5$ \\
\hline NGC 4339 & $112.9 \pm 3.7$ & 1 & $\ldots$ & $128.7 \pm 9.1$ & $128.7 \pm 9.1$ & 6 & $112.9 \pm 3.7$ \\
\hline NGC 4340 & $116.3 \pm 2.9$ & 1 & $\ldots$ & $101.1 \pm 8.5$ & $101.1 \pm 8.5$ & 6 & $116.3 \pm 2.9$ \\
\hline NGC 4346 & $\ldots$ & $\cdots$ & $150.5 \pm 11.1$ & $143.8 \pm 9.2$ & $146.5 \pm 7.1$ & & $146.5 \pm 7.1$ \\
\hline NGC 4350 & $180.5 \pm 7.2$ & 1 & $182.8 \pm 13.1$ & $201.6 \pm 10.2$ & $194.5 \pm 8.0$ & & $180.5 \pm 7.2$ \\
\hline NGC 4365 & $256.2 \pm 3.3$ & 1 & $249.1 \pm 18.5$ & $257.0 \pm 11.1$ & $254.9 \pm 9.5$ & & $256.2 \pm 3.3$ \\
\hline NGC 4369 & $\ldots$ & $\ldots$ & $\ldots$ & $71.6 \pm 9.1$ & $71.6 \pm 9.1$ & 6 & $71.6 \pm 9.1$ \\
\hline NGC 4371 & $134.6 \pm 4.5$ & 1 & $129.2 \pm 10.6$ & $126.8 \pm 9.1$ & $127.8 \pm 6.9$ & & $134.6 \pm 4.5$ \\
\hline NGC 4374 & $308 \pm 7$ & 2 & $274.5 \pm 20.7$ & $275.8 \pm 11.5$ & $275.5 \pm 10.1$ & & $308 \pm 7$ \\
\hline NGC 4378 & $197.8 \pm 9.6$ & 1 & $176.0 \pm 13.2$ & $201.3 \pm 10.1$ & $192.0 \pm 8.0$ & & $192.0 \pm 8.0$ \\
\hline NGC 4379 & $108.4 \pm 4.1$ & 1 & $110.4 \pm 11.8$ & $123.1 \pm 9.1$ & $118.4 \pm 7.2$ & & $108.4 \pm 4.1$ \\
\hline NGC 4380 & $62.2 \pm 18.2$ & 1 & $121.0 \pm 13.1$ & $62.9 \pm 8.4$ & $79.8 \pm 7.1$ & & $79.8 \pm 7.1$ \\
\hline NGC 4382 & $178.6 \pm 4.8$ & 1 & $163.9 \pm 13.9$ & $195.5 \pm 10.1$ & $184.6 \pm 8.2$ & & $178.6 \pm 4.8$ \\
\hline NGC 4388 & $115.2 \pm 17.1$ & 1 & $\ldots$ & $91.7 \pm 9.5$ & $91.7 \pm 9.5$ & 2 & $91.7 \pm 9.5$ \\
\hline NGC 4394 & $137.7 \pm 15.6$ & 1 & $\ldots$ & $115.5 \pm 8.9$ & $115.5 \pm 8.9$ & & $115.5 \pm 8.9$ \\
\hline NGC 4395 & $30 \pm$ & 13 & $\cdots$ & $\cdots$ & $\ldots$ & 2 & $30 \pm$ \\
\hline NGC 4405 & $\ldots$ & $\ldots$ & $\ldots$ & $\ldots$ & $\cdots$ & $1,6,7$ & $50.2 \pm 23.5$ \\
\hline NGC 4406 & $235.0 \pm 3.0$ & 1 & $225.2 \pm 16.7$ & $259.1 \pm 11.3$ & $248.5 \pm 9.4$ & & $235.0 \pm 3.0$ \\
\hline NGC 4414 & $117 \pm 4$ & 2 & $\ldots$ & $118.1 \pm 9.0$ & $118.1 \pm 9.0$ & 6 & $117 \pm 4$ \\
\hline NGC 4417 & $131.2 \pm 6.2$ & 1 & $131.7 \pm 11.1$ & $148.8 \pm 9.4$ & $141.7 \pm 7.2$ & & $131.2 \pm 6.2$ \\
\hline NGC 4419 & $99.2 \pm 3.2$ & 1 & $\ldots$ & $132.0 \pm 9.4$ & $132.0 \pm 9.4$ & 6 & $99.2 \pm 3.2$ \\
\hline NGC 4421 & $112.1 \pm 17.8$ & 1 & $\ldots$ & $55.8 \pm 8.3$ & $55.8 \pm 8.3$ & 6 & $55.8 \pm 8.3$ \\
\hline NGC 4424 & $\cdots$ & $\cdots$ & $\ldots$ & $56.8 \pm 9.2$ & $56.8 \pm 9.2$ & 2 & $56.8 \pm 9.2$ \\
\hline NGC 4429 & $192.5 \pm 7.5$ & 1 & $129.3 \pm 12.2$ & $176.6 \pm 9.7$ & $158.3 \pm 7.6$ & & $192.5 \pm 7.5$ \\
\hline NGC 4435 & $156.7 \pm 5.8$ & 1 & $129.7 \pm 14.0$ & $174.2 \pm 9.7$ & $159.8 \pm 8.0$ & & $156.7 \pm 5.8$ \\
\hline NGC 4438 & $\ldots$ & $\ldots$ & $131.2 \pm 10.8$ & $139.0 \pm 9.2$ & $135.7 \pm 7.0$ & & $135.7 \pm 7.0$ \\
\hline NGC 4442 & $186.7 \pm 8.7$ & 1 & $176.3 \pm 12.3$ & $194.4 \pm 10.1$ & $187.1 \pm 7.8$ & & $187.1 \pm 7.8$ \\
\hline NGC 4448 & $173.5 \pm 26.5$ & 1 & $\ldots$ & $119.8 \pm 9.0$ & $119.8 \pm 9.0$ & 6 & $119.8 \pm 9.0$ \\
\hline NGC 4449 & $\ldots$ & $\ldots$ & $\ldots$ & $17.8 \pm 9.1$ & $17.8 \pm 9.1$ & $2,7,12$ & $17.8 \pm 9.1$ \\
\hline NGC 4450 & $129.6 \pm 15.5$ & 1 & $133.0 \pm 10.6$ & $136.5 \pm 9.1$ & $135.0 \pm 6.9$ & & $135.0 \pm 6.9$ \\
\hline NGC 4457 & $95.9 \pm 15.1$ & 1 & $\ldots$ & $119.3 \pm 9.0$ & $119.3 \pm 9.0$ & 6 & $119.3 \pm 9.0$ \\
\hline NGC 4459 & $169.9 \pm 7.1$ & 1 & $156.5 \pm 14.1$ & $194.7 \pm 9.8$ & $182.3 \pm 8.0$ & & $169.9 \pm 7.1$ \\
\hline NGC 4460 & $\ldots$ & $\cdots$ & $\ldots$ & $39.8 \pm 8.9$ & $39.8 \pm 8.9$ & $2,7,12$ & $39.8 \pm 8.9$ \\
\hline NGC 4461 & $150.8 \pm 6.4$ & 1 & $143.4 \pm 11.3$ & $140.7 \pm 9.6$ & $141.8 \pm 7.3$ & & $150.8 \pm 6.4$ \\
\hline NGC 4469 & $\ldots$ & $\cdots$ & $109.6 \pm 11.5$ & $104.8 \pm 9.3$ & $106.7 \pm 7.2$ & & $106.7 \pm 7.2$ \\
\hline NGC 4470 & $\cdots$ & $\cdots$ & $\ldots$ & $89.9 \pm 9.0$ & $89.9 \pm 9.0$ & 6 & $89.9 \pm 9.0$ \\
\hline NGC 4472 & $291.1 \pm 2.9$ & 1 & $279.7 \pm 21.0$ & $230.2 \pm 10.6$ & $240.3 \pm 9.5$ & & $291.1 \pm 2.9$ \\
\hline NGC 4473 & $179.3 \pm 2.9$ & 1 & $160.3 \pm 12.3$ & $196.2 \pm 10.1$ & $181.7 \pm 7.8$ & & $179.3 \pm 2.9$ \\
\hline NGC 4477 & $186.2 \pm 15.2$ & 1 & $162.5 \pm 12.0$ & $186.4 \pm 9.8$ & $176.8 \pm 7.6$ & & $176.8 \pm 7.6$ \\
\hline NGC 4478 & $137.4 \pm 2.3$ & 1 & $114.3 \pm 11.3$ & $161.4 \pm 9.5$ & $141.9 \pm 7.3$ & & $137.4 \pm 2.3$ \\
\hline NGC 4485 & $\cdots$ & $\cdots$ & $\ldots$ & $\ldots$ & $\ldots$ & $1,2,7$ & $52.2 \pm 24.4$ \\
\hline NGC 4486 & $332.2 \pm 4.9$ & 1 & $354.6 \pm 38.0$ & $349.8 \pm 13.0$ & $350.3 \pm 12.3$ & & $332.2 \pm 4.9$ \\
\hline NGC 4490 & $\ldots$ & $\ldots$ & $\ldots$ & $45.1 \pm 9.0$ & $45.1 \pm 9.0$ & $2,7,12$ & $45.1 \pm 9.0$ \\
\hline NGC 4494 & $145 \pm 3$ & 2 & $126.3 \pm 10.5$ & $168.5 \pm 9.5$ & $149.5 \pm 7.0$ & & $145 \pm 3$ \\
\hline
\end{tabular}


Table 1

(Continued)

\begin{tabular}{|c|c|c|c|c|c|c|c|}
\hline \multirow[t]{2}{*}{ Galaxy } & \multicolumn{2}{|c|}{ Literature } & \multicolumn{4}{|c|}{ Palomar } & \multirow{2}{*}{$\begin{array}{c}\text { Adopted } \\
\begin{array}{c}\sigma \\
\left(\mathrm{km} \mathrm{s}^{-1}\right)\end{array}\end{array}$} \\
\hline & $\begin{array}{c}\sigma \\
\left(\mathrm{km} \mathrm{s}^{-1}\right) \\
\end{array}$ & Reference & $\begin{array}{c}\sigma_{\text {blue }} \\
\left(\mathrm{km} \mathrm{s}^{-1}\right)\end{array}$ & $\begin{array}{c}\sigma_{\text {red }} \\
\left(\mathrm{km} \mathrm{s}^{-1}\right)\end{array}$ & $\begin{array}{c}\sigma_{\text {final }} \\
\left(\mathrm{km} \mathrm{s}^{-1}\right)\end{array}$ & Notes & \\
\hline NGC 4496A & $\ldots$ & $\ldots$ & $\ldots$ & $\ldots$ & $\ldots$ & 1,2 & $74.9 \pm 35.1$ \\
\hline NGC 4496B & $\ldots$ & $\ldots$ & $132.9 \pm 22.6$ & $100.6 \pm 9.7$ & $105.6 \pm 8.9$ & & $105.6 \pm 8.9$ \\
\hline NGC 4501 & $160.9 \pm 12.8$ & 1 & $198.0 \pm 11.9$ & $147.7 \pm 9.3$ & $166.8 \pm 7.3$ & & $166.9 \pm 7.3$ \\
\hline NGC 4503 & $110.8 \pm 25.4$ & 1 & $142.6 \pm 11.3$ & $149.1 \pm 9.4$ & $146.4 \pm 7.2$ & & $146.4 \pm 7.2$ \\
\hline NGC 4517 & $\ldots$ & $\ldots$ & $\ldots$ & $43.8 \pm 8.5$ & $43.8 \pm 8.5$ & $7,8,12$ & $43.8 \pm 8.5$ \\
\hline NGC 4526 & $263.7 \pm 18.9$ & 1 & $204.2 \pm 16.2$ & $216.3 \pm 10.3$ & $212.8 \pm 8.7$ & & $212.8 \pm 8.7$ \\
\hline NGC 4527 & $210.7 \pm 10.2$ & 1 & $131.3 \pm 15.7$ & $136.7 \pm 9.2$ & $135.3 \pm 7.9$ & & $135.3 \pm 7.9$ \\
\hline NGC 4532 & $\ldots$ & $\ldots$ & $\ldots$ & $\ldots$ & $\ldots$ & 1,2 & $<70.9$ \\
\hline NGC 4535 & $\ldots$ & $\ldots$ & $\ldots$ & $102.5 \pm 10.2$ & $102.5 \pm 10.2$ & 8 & $102.5 \pm 10.2$ \\
\hline NGC 4536 & $85 \pm 1$ & 6 & $109.0 \pm 16.5$ & $101.9 \pm 9.5$ & $103.7 \pm 8.2$ & & $85 \pm 1$ \\
\hline NGC 4548 & $144.3 \pm 15$ & 1 & $\ldots$ & $113.4 \pm 8.9$ & $113.4 \pm 8.9$ & 6 & $113.4 \pm 8.9$ \\
\hline NGC 4550 & $90.9 \pm 4.4$ & 1 & $\ldots$ & $63.3 \pm 8.9$ & $63.3 \pm 8.9$ & 6 & $90.9 \pm 4.4$ \\
\hline NGC 4552 & $252.4 \pm 3.4$ & 1 & $247.4 \pm 17.2$ & $278.5 \pm 11.6$ & $268.8 \pm 9.6$ & & $252.4 \pm 3.4$ \\
\hline NGC 4559 & $\ldots$ & $\cdots$ & $\ldots$ & $49.2 \pm 8.6$ & $49.2 \pm 8.6$ & 6 & $49.2 \pm 8.6$ \\
\hline NGC 4564 & $157.4 \pm 3.1$ & 1 & $163.5 \pm 11.8$ & $159.3 \pm 9.5$ & $161.0 \pm 7.4$ & & $157.4 \pm 3.1$ \\
\hline NGC 4565 & $136.0 \pm 6.3$ & 1 & $173.8 \pm 11.6$ & $158.4 \pm 9.4$ & $164.5 \pm 7.3$ & & $136.0 \pm 6.3$ \\
\hline NGC 4567 & $\ldots$ & $\cdots$ & $\ldots$ & $66.0 \pm 9.1$ & $66.0 \pm 9.1$ & 6 & $66.0 \pm 9.1$ \\
\hline NGC 4568 & $\ldots$ & $\ldots$ & $\ldots$ & $88.3 \pm 9.4$ & $88.3 \pm 9.4$ & 6 & $88.3 \pm 9.4$ \\
\hline NGC 4569 & $136 \pm 3$ & 2 & $112.1 \pm 29.2$ & $173.8 \pm 9.7$ & $167.7 \pm 9.2$ & & $136 \pm 3$ \\
\hline NGC 4570 & $187.9 \pm 8.5$ & 1 & $188.7 \pm 12.1$ & $203.6 \pm 10.2$ & $197.4 \pm 7.8$ & & $197.4 \pm 7.8$ \\
\hline NGC 4578 & $120.4 \pm 9.6$ & 1 & $108.4 \pm 10.1$ & $115.5 \pm 9.0$ & $112.4 \pm 6.7$ & & $112.4 \pm 6.7$ \\
\hline NGC 4579 & $165 \pm 4$ & 2 & $161.9 \pm 12.3$ & $209.1 \pm 10.8$ & $188.6 \pm 8.1$ & & $165 \pm 4$ \\
\hline NGC 4589 & $224.3 \pm 5.6$ & 1 & $228.6 \pm 16.4$ & $225.5 \pm 10.5$ & $226.4 \pm 8.8$ & & $224.3 \pm 5.6$ \\
\hline NGC 4594 & $241.1 \pm 4.4$ & 1 & $250.8 \pm 16.4$ & $223.2 \pm 10.5$ & $231.2 \pm 8.8$ & & $241.1 \pm 4.4$ \\
\hline NGC 4596 & $148.8 \pm 2.9$ & 1 & $133.5 \pm 11.0$ & $155.7 \pm 10.1$ & $145.5 \pm 7.4$ & & $148.8 \pm 2.9$ \\
\hline NGC 4605 & $\ldots$ & $\cdots$ & $\ldots$ & $26.1 \pm 9.3$ & $26.1 \pm 9.3$ & $6,7,12$ & $26.1 \pm 9.3$ \\
\hline NGC 4608 & $160.8 \pm 14.2$ & 1 & $133.7 \pm 11.2$ & $143.0 \pm 9.3$ & $139.2 \pm 7.2$ & & $139.2 \pm 7.2$ \\
\hline NGC 4612 & $63.2 \pm 4.2$ & 1 & $\ldots$ & $80.8 \pm 8.5$ & $80.8 \pm 8.5$ & 6 & $63.2 \pm 4.2$ \\
\hline NGC 4618 & $\ldots$ & $\cdots$ & $\cdots$ & $\ldots$ & & 1,2 & $<54.6$ \\
\hline NGC 4621 & $225.2 \pm 3.3$ & 1 & $237.2 \pm 15.5$ & $270.2 \pm 11.3$ & $258.7 \pm 9.1$ & & $225.2 \pm 3.3$ \\
\hline NGC 4631 & $\ldots$ & $\ldots$ & $\ldots$ & $\ldots$ & $\ldots$ & 1,2 & $<71.9$ \\
\hline NGC 4636 & $202.7 \pm 3.4$ & 1 & $219.8 \pm 15.2$ & $221.9 \pm 10.5$ & $221.2 \pm 8.6$ & & $202.7 \pm 3.4$ \\
\hline NGC 4638 & $121.9 \pm 4.0$ & 1 & $\ldots$ & $120.9 \pm 9.0$ & $120.9 \pm 9.0$ & 6 & $121.9 \pm 4.0$ \\
\hline NGC 4639 & $96 \pm 4$ & 2 & $\ldots$ & $96.2 \pm 9.1$ & $96.2 \pm 9.1$ & 6 & $96 \pm 4$ \\
\hline NGC 4643 & $163 \pm 7.9$ & 1 & $148.8 \pm 11.6$ & $140.0 \pm 10.0$ & $143.8 \pm 7.6$ & & $143.8 \pm 7.6$ \\
\hline NGC 4647 & $97.6 \pm 38.8$ & 1 & $\ldots$ & $17.5 \pm 8.8$ & $17.5 \pm 8.8$ & $6,7,12$ & $17.5 \pm 8.8$ \\
\hline NGC 4648 & $215.9 \pm 10.1$ & 1 & $237.8 \pm 15.8$ & $218.6 \pm 10.5$ & $224.5 \pm 8.7$ & & $224.5 \pm 8.7$ \\
\hline NGC 4649 & $335.3 \pm 4.5$ & 1 & $349.1 \pm 23.6$ & $376.5 \pm 13.5$ & $369.7 \pm 11.7$ & & $335.3 \pm 4.5$ \\
\hline NGC 4651 & $\ldots$ & $\ldots$ & $\ldots$ & $100.9 \pm 8.7$ & $100.9 \pm 8.7$ & 6 & $100.9 \pm 8.7$ \\
\hline NGC 4654 & $\ldots$ & $\cdots$ & $\cdots$ & $47.7 \pm 9.0$ & $47.7 \pm 9.0$ & 8 & $47.7 \pm 9.0$ \\
\hline NGC 4656 & $\ldots$ & $\ldots$ & $\ldots$ & $\ldots$ & $\ldots$ & $1,8,9$ & $70.4 \pm 32.9$ \\
\hline NGC 4660 & $188.5 \pm 3.4$ & 1 & $223.1 \pm 15.2$ & $213.4 \pm 10.3$ & $216.5 \pm 8.5$ & & $188.5 \pm 3.4$ \\
\hline NGC 4665 & $\ldots$ & $\cdots$ & $168.0 \pm 12.0$ & $129.7 \pm 9.1$ & $143.7 \pm 7.3$ & & $143.7 \pm 7.3$ \\
\hline NGC 4688 & $\ldots$ & $\ldots$ & $\ldots$ & $73.0 \pm 8.8$ & $73.0 \pm 8.8$ & 6 & $73.0 \pm 8.8$ \\
\hline NGC 4689 & $\ldots$ & $\ldots$ & $\ldots$ & $41.4 \pm 8.4$ & $41.4 \pm 8.4$ & $7,8,12$ & $41.4 \pm 8.4$ \\
\hline NGC 4694 & $61.3 \pm 6.5$ & 1 & $\ldots$ & $\ldots$ & $\ldots$ & 2,7 & $61.3 \pm 6.5$ \\
\hline NGC 4698 & $132.7 \pm 8.6$ & 1 & $140.3 \pm 11.6$ & $154.1 \pm 9.5$ & $148.6 \pm 7.3$ & & $148.6 \pm 7.3$ \\
\hline NGC 4710 & $142.2 \pm 9.5$ & 1 & $\ldots$ & $109.6 \pm 9.5$ & $109.6 \pm 9.5$ & 6 & $109.6 \pm 9.5$ \\
\hline NGC 4713 & $\ldots$ & $\ldots$ & $\ldots$ & $23.2 \pm 8.9$ & $23.2 \pm 8.9$ & $6,7,12$ & $23.2 \pm 8.9$ \\
\hline NGC 4725 & $140 \pm 3$ & 2 & $141.0 \pm 10.3$ & $130.8 \pm 9.1$ & $135.3 \pm 6.8$ & & $140 \pm 3$ \\
\hline NGC 4736 & $112 \pm 3$ & 2 & $125.0 \pm 11.9$ & $126.3 \pm 9.8$ & $125.8 \pm 7.6$ & & $112 \pm 3$ \\
\hline NGC 4750 & $\ldots$ & $\cdots$ & $131.4 \pm 11.0$ & $139.2 \pm 9.2$ & $136.0 \pm 7.1$ & & $136.0 \pm 7.1$ \\
\hline NGC 4754 & $184.9 \pm 4.3$ & 1 & $173.5 \pm 12.3$ & $200.7 \pm 10.1$ & $189.7 \pm 7.8$ & & $184.9 \pm 4.3$ \\
\hline NGC 4762 & $147.1 \pm 9.4$ & 1 & $151.9 \pm 11.3$ & $151.7 \pm 9.4$ & $151.8 \pm 7.2$ & & $151.8 \pm 7.2$ \\
\hline NGC 4772 & $\ldots$ & $\cdots$ & $152.3 \pm 13.2$ & $146.3 \pm 9.4$ & $148.3 \pm 7.7$ & & $148.3 \pm 7.7$ \\
\hline NGC 4793 & $\ldots$ & $\ldots$ & $\ldots$ & $26.6 \pm 8.5$ & $26.6 \pm 8.5$ & $2,7,12$ & $26.6 \pm 8.5$ \\
\hline NGC 4800 & $111 \pm 4$ & 2 & $117.8 \pm 12.4$ & $99.1 \pm 8.7$ & $105.3 \pm 7.1$ & & $111 \pm 4$ \\
\hline NGC 4826 & $96 \pm 3$ & 2 & $147.3 \pm 11.9$ & $110.5 \pm 8.9$ & $123.7 \pm 7.1$ & & $96 \pm 3$ \\
\hline NGC 4845 & $\ldots$ & $\ldots$ & $\ldots$ & $133.9 \pm 9.3$ & $133.9 \pm 9.3$ & 6 & $133.9 \pm 9.3$ \\
\hline NGC 4866 & $210.1 \pm 9.7$ & 1 & $183.3 \pm 12.8$ & $227.1 \pm 10.5$ & $209.5 \pm 8.1$ & & $209.5 \pm 8.1$ \\
\hline NGC 4900 & $\ldots$ & $\cdots$ & $\ldots$ & $58.6 \pm 9.0$ & $58.6 \pm 9.0$ & 6 & $58.6 \pm 9.0$ \\
\hline NGC 4914 & $223.6 \pm 25.1$ & 1 & $229.8 \pm 16.9$ & $222.7 \pm 10.5$ & $224.7 \pm 8.9$ & & $224.7 \pm 8.9$ \\
\hline NGC 5005 & $154 \pm 10$ & 6 & $178.9 \pm 14.9$ & $169.2 \pm 9.6$ & $172.0 \pm 8.1$ & & $172.0 \pm 8.1$ \\
\hline NGC 5012 & $\ldots$ & $\ldots$ & $\ldots$ & $141.4 \pm 9.2$ & $141.4 \pm 9.2$ & 6 & $141.4 \pm 9.2$ \\
\hline
\end{tabular}


Table 1

(Continued)

\begin{tabular}{|c|c|c|c|c|c|c|c|}
\hline \multirow[t]{2}{*}{ Galaxy } & \multicolumn{2}{|c|}{ Literature } & \multicolumn{4}{|c|}{ Palomar } & \multirow{2}{*}{$\begin{array}{c}\text { Adopted } \\
\sigma \\
\left(\mathrm{km} \mathrm{s}^{-1}\right)\end{array}$} \\
\hline & $\begin{array}{c}\sigma \\
\left(\mathrm{km} \mathrm{s}^{-1}\right) \\
\end{array}$ & Reference & $\begin{array}{c}\begin{array}{c}\sigma_{\text {blue }} \\
\left(\mathrm{km} \mathrm{s}^{-1}\right)\end{array} \\
\end{array}$ & $\begin{array}{c}\sigma_{\text {red }} \\
\left(\mathrm{km} \mathrm{s}^{-1}\right)\end{array}$ & $\begin{array}{c}\sigma_{\text {final }} \\
\left(\mathrm{km} \mathrm{s}^{-1}\right)\end{array}$ & Notes & \\
\hline NGC 5033 & $151 \pm 4$ & 2 & $154.1 \pm 13.9$ & $130.3 \pm 9.4$ & $137.8 \pm 7.8$ & & $151 \pm 4$ \\
\hline NGC 5055 & $117 \pm 6$ & 6 & $\ldots$ & $106.3 \pm 8.9$ & $106.3 \pm 8.9$ & 6 & $117 \pm 6$ \\
\hline NGC 5077 & $254.6 \pm 7.9$ & 1 & $278.7 \pm 19.7$ & $253.1 \pm 11.0$ & $259.2 \pm 9.6$ & & $254.6 \pm 7.9$ \\
\hline NGC 5112 & $\cdots$ & $\cdots$ & $\cdots$ & $\cdots$ & $\ldots$ & $1,6,7$ & $<60.8$ \\
\hline NGC 5147 & $\ldots$ & $\ldots$ & $\ldots$ & $52.3 \pm 8.8$ & $52.3 \pm 8.8$ & 6 & $52.3 \pm 8.8$ \\
\hline NGC 5194 & $96.0 \pm 8.7$ & 1 & $\ldots$ & $76.3 \pm 9.1$ & $76.3 \pm 9.1$ & 6 & $96.0 \pm 8.7$ \\
\hline NGC 5195 & $146.8 \pm 15.1$ & 1 & $113.8 \pm 14.9$ & $129.4 \pm 9.7$ & $124.8 \pm 8.1$ & & $124.8 \pm 8.1$ \\
\hline NGC 5204 & $\ldots$ & $\ldots$ & $\ldots$ & $\ldots$ & $\ldots$ & 1,2 & $39.9 \pm 18.7$ \\
\hline NGC 5248 & $\cdots$ & $\cdots$ & $\cdots$ & $99.4 \pm 9.2$ & $99.4 \pm 9.2$ & 6 & $99.4 \pm 9.2$ \\
\hline NGC 5273 & $71 \pm 4$ & 2 & $\ldots$ & $76.0 \pm 8.8$ & $76.0 \pm 8.8$ & 6,8 & $71 \pm 4$ \\
\hline NGC 5297 & $118.9 \pm 20.3$ & 1 & $109.1 \pm 10.6$ & $61.3 \pm 9.0$ & $81.3 \pm 6.9$ & & $61.3 \pm 9.0$ \\
\hline NGC 5300 & $\cdots$ & $\cdots$ & $\ldots$ & $19.8 \pm 8.4$ & $19.8 \pm 8.4$ & $2,7,12$ & $19.8 \pm 8.4$ \\
\hline NGC 5308 & $210.5 \pm 12.1$ & 1 & $214.1 \pm 17.3$ & $263.9 \pm 11.3$ & $249.0 \pm 9.5$ & & $249.0 \pm 9.5$ \\
\hline NGC 5322 & $232.2 \pm 4.4$ & 1 & $238.0 \pm 17.0$ & $245.3 \pm 13.1$ & $242.6 \pm 10.4$ & & $232.2 \pm 4.4$ \\
\hline NGC 5353 & $286.4 \pm 5.4$ & 1 & $314.9 \pm 20.7$ & $289.0 \pm 11.6$ & $295.2 \pm 10.1$ & & $286.4 \pm 5.4$ \\
\hline NGC 5354 & $217.4 \pm 6.4$ & 1 & $176.0 \pm 14.6$ & $231.3 \pm 10.5$ & $212.4 \pm 8.5$ & & $217.4 \pm 6.4$ \\
\hline NGC 5363 & $195.5 \pm 14.7$ & 1 & $202.9 \pm 14.8$ & $240.6 \pm 10.9$ & $227.3 \pm 8.8$ & & $227.3 \pm 8.8$ \\
\hline NGC 5364 & $91.4 \pm 51.9$ & 1 & $\ldots$ & $22.2 \pm 8.9$ & $22.2 \pm 8.9$ & $6,7,12$ & $22.2 \pm 8.9$ \\
\hline NGC 5371 & $\cdots$ & $\ldots$ & $187.4 \pm 13.3$ & $175.7 \pm 9.7$ & $179.8 \pm 7.8$ & & $179.8 \pm 7.8$ \\
\hline NGC 5377 & $\ldots$ & $\ldots$ & $118.1 \pm 14.8$ & $193.3 \pm 10.0$ & $169.7 \pm 8.3$ & & $169.7 \pm 8.3$ \\
\hline NGC 5383 & $\cdots$ & $\cdots$ & $\ldots$ & $96.5 \pm 8.7$ & $96.5 \pm 8.7$ & 6 & $96.5 \pm 8.7$ \\
\hline NGC 5395 & $\cdots$ & $\cdots$ & $161.6 \pm 13.2$ & $135.5 \pm 10.4$ & $145.5 \pm 8.2$ & & $145.5 \pm 8.2$ \\
\hline NGC 5448 & $\ldots$ & $\ldots$ & $121.4 \pm 14.9$ & $125.8 \pm 9.7$ & $124.5 \pm 8.1$ & & $124.5 \pm 8.1$ \\
\hline NGC 5457 & $72.9 \pm 16.6$ & 1 & $\ldots$ & $23.6 \pm 8.7$ & $23.6 \pm 8.7$ & $7,8,12$ & $23.6 \pm 8.7$ \\
\hline NGC 5473 & $221.6 \pm 8.9$ & 1 & $193.4 \pm 15.6$ & $233.3 \pm 10.7$ & $220.5 \pm 8.8$ & & $220.5 \pm 8.8$ \\
\hline NGC 5474 & & $\ldots$ & $\ldots$ & $29.0 \pm 8.6$ & $29.0 \pm 8.6$ & $7,8,12$ & $29.0 \pm 8.6$ \\
\hline NGC 5485 & $159.1 \pm 23.9$ & 1 & $179.2 \pm 14.6$ & $221.8 \pm 10.4$ & $207.5 \pm 8.5$ & & $207.5 \pm 8.5$ \\
\hline NGC 5523 & $\ldots$ & $\ldots$ & $\ldots$ & $30.1 \pm 8.5$ & $30.1 \pm 8.5$ & $6,7,12$ & $30.1 \pm 8.5$ \\
\hline NGC 5548 & $291 \pm 12$ & 8 & $\ldots$ & $\ldots$ & $\ldots$ & 2 & $291 \pm 12$ \\
\hline NGC 5557 & $250.9 \pm 12.1$ & 1 & $283.2 \pm 19.5$ & $299.8 \pm 11.9$ & $295.3 \pm 10.2$ & & $295.3 \pm 10.2$ \\
\hline NGC 5566 & $\ldots$ & $\ldots$ & $147.9 \pm 11.8$ & $167.0 \pm 9.5$ & $159.5 \pm 7.4$ & & $159.5 \pm 7.4$ \\
\hline NGC 5576 & $182.3 \pm 7.3$ & 1 & $214.4 \pm 17.9$ & $218.2 \pm 10.5$ & $217.2 \pm 9.1$ & & $182.3 \pm 7.3$ \\
\hline NGC 5585 & $42 \pm$ & 4 & $\ldots$ & $\ldots$ & $\ldots$ & 8,9 & $42 \pm$ \\
\hline NGC 5631 & $168.2 \pm 10.8$ & 1 & $173.6 \pm 13.1$ & $164.5 \pm 10.5$ & $168.1 \pm 8.2$ & & $168.1 \pm 8.2$ \\
\hline NGC 5638 & $165.0 \pm 3.5$ & 1 & $142.0 \pm 10.8$ & $178.7 \pm 9.8$ & $162.1 \pm 7.3$ & & $165.0 \pm 3.5$ \\
\hline NGC 5656 & $\cdots$ & $\cdots$ & $\cdots$ & $116.7 \pm 9.0$ & $116.7 \pm 9.0$ & 6 & $116.7 \pm 9.0$ \\
\hline NGC 5660 & $\cdots$ & $\ldots$ & $\ldots$ & $60.7 \pm 9.4$ & $60.7 \pm 9.4$ & 6 & $60.7 \pm 9.4$ \\
\hline NGC 5668 & $53 \pm$ & 4 & $\cdots$ & $\ldots$ & $\ldots$ & 6,9 & $53 \pm$ \\
\hline NGC 5669 & $\cdots$ & $\cdots$ & $\cdots$ & $\cdots$ & $\ldots$ & 1,2 & $32.4 \pm 15.2$ \\
\hline NGC 5676 & $117.8 \pm 15.5$ & 1 & $\ldots$ & $116.7 \pm 8.9$ & $116.7 \pm 8.9$ & 6 & $116.7 \pm 8.9$ \\
\hline NGC 5678 & $103 \pm$ & 4 & $\ldots$ & $132.8 \pm 10.2$ & $132.8 \pm 10.2$ & 6 & $132.8 \pm 10.2$ \\
\hline NGC 5690 & $\ldots$ & $\ldots$ & $\ldots$ & $\ldots$ & $\ldots$ & 1,2 & $<64.3$ \\
\hline NGC 5701 & $115.2 \pm 15.4$ & 1 & $131.7 \pm 11.6$ & $119.2 \pm 9.7$ & $124.3 \pm 7.4$ & & $124.3 \pm 7.4$ \\
\hline NGC 5746 & $182.5 \pm 9.9$ & 1 & $229.7 \pm 15.1$ & $187.4 \pm 9.9$ & $200.1 \pm 8.3$ & & $200.1 \pm 8.3$ \\
\hline NGC 5775 & $\cdots$ & $\ldots$ & $120.0 \pm 12.4$ & $89.5 \pm 9.2$ & $100.3 \pm 7.4$ & & $100.3 \pm 7.4$ \\
\hline NGC 5806 & $\ldots$ & $\ldots$ & $\ldots$ & $124.7 \pm 9.1$ & $124.7 \pm 9.1$ & 6 & $124.7 \pm 9.1$ \\
\hline NGC 5813 & $238.7 \pm 4.8$ & 1 & $232.9 \pm 16.2$ & $266.7 \pm 11.3$ & $255.6 \pm 9.3$ & & $238.7 \pm 4.8$ \\
\hline NGC 5831 & $164.4 \pm 4.7$ & 1 & $161.4 \pm 11.7$ & $181.1 \pm 9.7$ & $173.1 \pm 7.5$ & & $164.4 \pm 4.7$ \\
\hline NGC 5838 & $265.7 \pm 9.3$ & 1 & $268.7 \pm 20.2$ & $328.8 \pm 12.6$ & $312.0 \pm 10.7$ & & $265.7 \pm 9.3$ \\
\hline NGC 5846 & $236.8 \pm 4.4$ & 1 & $225.3 \pm 15.8$ & $276.2 \pm 10.3$ & $261.0 \pm 8.6$ & & $236.8 \pm 4.4$ \\
\hline NGC 5850 & $\cdots$ & $\cdots$ & $147.1 \pm 10.5$ & $135.4 \pm 9.1$ & $140.4 \pm 6.9$ & & $140.4 \pm 6.9$ \\
\hline NGC 5866 & $158.9 \pm 9.8$ & 1 & $144.7 \pm 13.7$ & $181.8 \pm 9.9$ & $169.1 \pm 8.0$ & & $169.1 \pm 8.0$ \\
\hline NGC 5879 & $73.9 \pm 8.7$ & 1 & $\ldots$ & $90.4 \pm 8.9$ & $90.4 \pm 8.9$ & 6 & $73.9 \pm 8.7$ \\
\hline NGC 5905 & $\ldots$ & $\ldots$ & $185.7 \pm 25.0$ & $172.9 \pm 9.7$ & $174.6 \pm 9.0$ & & $174.6 \pm 9.0$ \\
\hline NGC 5907 & $\ldots$ & $\ldots$ & $108.7 \pm 11.6$ & $127.2 \pm 9.0$ & $120.2 \pm 7.1$ & & $120.2 \pm 7.1$ \\
\hline NGC 5921 & $\ldots$ & $\ldots$ & $\ldots$ & $83.9 \pm 9.2$ & $83.9 \pm 9.2$ & 6 & $83.9 \pm 9.2$ \\
\hline NGC 5962 & $\cdots$ & $\cdots$ & $\ldots$ & $106.3 \pm 9.5$ & $106.3 \pm 9.5$ & 6 & $106.3 \pm 9.5$ \\
\hline NGC 5970 & $\ldots$ & $\ldots$ & $\ldots$ & $116.3 \pm 9.0$ & $116.3 \pm 9.0$ & 6 & $116.3 \pm 9.0$ \\
\hline NGC 5982 & $239.4 \pm 5.2$ & 1 & $272.4 \pm 20.1$ & $250.5 \pm 11.0$ & $255.5 \pm 9.6$ & & $239.4 \pm 5.2$ \\
\hline NGC 5985 & $\ldots$ & $\ldots$ & $153.8 \pm 12.3$ & $159.8 \pm 9.5$ & $157.6 \pm 7.5$ & & $157.6 \pm 7.5$ \\
\hline NGC 6015 & $\cdots$ & $\ldots$ & $\ldots$ & $43.5 \pm 8.8$ & $43.5 \pm 8.8$ & $6,7,12$ & $43.5 \pm 8.8$ \\
\hline NGC 6070 & $\ldots$ & $\ldots$ & $\ldots$ & $93.7 \pm 9.1$ & $93.7 \pm 9.1$ & 6 & $93.7 \pm 9.1$ \\
\hline NGC 6140 & $34.4 \pm 12.4$ & 1 & $\ldots$ & $49.4 \pm 8.9$ & $49.4 \pm 8.9$ & 2 & $49.4 \pm 8.9$ \\
\hline NGC 6181 & $\ldots$ & $\ldots$ & $\ldots$ & $125.2 \pm 9.0$ & $125.2 \pm 9.0$ & 6 & $125.2 \pm 9.0$ \\
\hline
\end{tabular}


Table 1

(Continued)

\begin{tabular}{|c|c|c|c|c|c|c|c|}
\hline \multirow[t]{2}{*}{ Galaxy } & \multicolumn{2}{|c|}{ Literature } & \multicolumn{4}{|c|}{ Palomar } & \multirow{2}{*}{$\begin{array}{c}\text { Adopted } \\
\sigma \\
\left(\mathrm{km} \mathrm{s}^{-1}\right)\end{array}$} \\
\hline & $\begin{array}{c}\sigma \\
\left(\mathrm{km} \mathrm{s}^{-1}\right)\end{array}$ & Reference & $\begin{array}{c}\sigma_{\text {blue }} \\
\left(\mathrm{km} \mathrm{s}^{-1}\right)\end{array}$ & $\begin{array}{c}\sigma_{\text {red }} \\
\left(\mathrm{km} \mathrm{s}^{-1}\right)\end{array}$ & $\begin{array}{c}\sigma_{\text {final }} \\
\left(\mathrm{km} \mathrm{s}^{-1}\right)\end{array}$ & Notes & \\
\hline NGC 6207 & $\ldots$ & $\ldots$ & $\ldots$ & $92.1 \pm 10.0$ & $92.1 \pm 10.0$ & 2 & $92.1 \pm 10.0$ \\
\hline NGC 6217 & $134.5 \pm 19.6$ & 1 & $\cdots$ & $70.3 \pm 10.0$ & $70.3 \pm 10.0$ & 8 & $70.3 \pm 10.0$ \\
\hline NGC 6236 & $\ldots$ & $\ldots$ & $\ldots$ & $\cdots$ & $\cdots$ & 1,2 & $46.1 \pm 21.6$ \\
\hline NGC 6340 & $143.9 \pm 5.6$ & 1 & $130.1 \pm 10.5$ & $147.9 \pm 9.2$ & $140.2 \pm 6.9$ & & $143.9 \pm 5.6$ \\
\hline NGC 6384 & $124.3 \pm 7.1$ & 1 & $111.2 \pm 10.9$ & $135.4 \pm 9.9$ & $124.5 \pm 7.3$ & & $124.3 \pm 7.1$ \\
\hline NGC 6412 & $29.8 \pm 23.1$ & 1 & $\cdots$ & $49.9 \pm 9.0$ & $49.9 \pm 9.0$ & 8 & $49.9 \pm 9.0$ \\
\hline NGC 6482 & $310.4 \pm 11.5$ & 1 & $\ldots$ & $337.0 \pm 13.0$ & $337.0 \pm 13.0$ & 8 & $310.4 \pm 11.5$ \\
\hline NGC 6500 & $214 \pm 6$ & 2 & $220.3 \pm 17.1$ & $212.0 \pm 10.3$ & $214.2 \pm 8.8$ & & $214 \pm 6$ \\
\hline NGC 6501 & $215.9 \pm 12.3$ & 1 & $\cdots$ & $235.0 \pm 10.7$ & $235.0 \pm 10.7$ & 5 & $235.0 \pm 10.7$ \\
\hline NGC 6503 & $46 \pm 3$ & 2 & $\ldots$ & $\ldots$ & $\ldots$ & 5,7 & $46 \pm 3$ \\
\hline NGC 6643 & $72.3 \pm 23.2$ & 1 & $\cdots$ & $95.4 \pm 9.2$ & $95.4 \pm 9.2$ & 6 & $95.4 \pm 9.2$ \\
\hline NGC 6654 & $\ldots$ & $\ldots$ & $175.4 \pm 12.3$ & $170.2 \pm 9.6$ & $172.2 \pm 7.6$ & & $172.2 \pm 7.6$ \\
\hline NGC 6689 & $26 \pm 11$ & 1 & $\cdots$ & $\ldots$ & $\ldots$ & 2,7 & $26 \pm 11$ \\
\hline NGC 6702 & $173.6 \pm 4.9$ & 1 & $\cdots$ & $169.6 \pm 9.7$ & $169.6 \pm 9.7$ & 5 & $173.6 \pm 4.9$ \\
\hline NGC 6703 & $179.9 \pm 3.7$ & 1 & $185.2 \pm 13.4$ & $191.4 \pm 10.0$ & $189.2 \pm 8.0$ & & $179.9 \pm 3.7$ \\
\hline NGC 6946 & $\cdots$ & $\cdots$ & $\ldots$ & $55.8 \pm 9.4$ & $55.8 \pm 9.4$ & 2 & $55.8 \pm 9.4$ \\
\hline NGC 6951 & $97.9 \pm 10.1$ & 1 & $\cdots$ & $127.8 \pm 9.1$ & $127.8 \pm 9.1$ & 6 & $127.8 \pm 9.1$ \\
\hline NGC 7080 & $\cdots$ & $\cdots$ & $\cdots$ & $95.3 \pm 9.4$ & $95.3 \pm 9.4$ & 5 & $95.3 \pm 9.4$ \\
\hline NGC 7177 & $124.1 \pm 3.7$ & 1 & $128.8 \pm 12.5$ & $137.7 \pm 9.2$ & $134.6 \pm 7.4$ & & $124.1 \pm 3.7$ \\
\hline NGC 7217 & $127.0 \pm 10.1$ & 1 & $155.0 \pm 12.7$ & $134.4 \pm 9.1$ & $141.4 \pm 7.4$ & & $141.4 \pm 7.4$ \\
\hline NGC 7331 & $137.2 \pm 3.5$ & 1 & $144.3 \pm 12.7$ & $131.6 \pm 9.1$ & $135.9 \pm 7.4$ & & $137.2 \pm 3.5$ \\
\hline NGC 7332 & $124.1 \pm 3.5$ & 1 & $131.3 \pm 11.7$ & $155.2 \pm 9.4$ & $145.8 \pm 7.3$ & & $124.1 \pm 3.5$ \\
\hline NGC 7448 & $56.1 \pm 14.8$ & 1 & $\cdots$ & $77.6 \pm 9.1$ & $77.6 \pm 9.1$ & 6 & $77.6 \pm 9.1$ \\
\hline NGC 7457 & $69.4 \pm 4.2$ & 1 & $\cdots$ & $67.1 \pm 8.5$ & $67.1 \pm 8.5$ & 6 & $69.4 \pm 4.2$ \\
\hline NGC 7479 & $109 \pm 11$ & 1 & $151.6 \pm 19.5$ & $155.4 \pm 9.4$ & $154.7 \pm 8.5$ & & $154.7 \pm 8.5$ \\
\hline NGC 7619 & $322.0 \pm 5.8$ & 1 & $315.9 \pm 22.1$ & $336.2 \pm 12.7$ & $331.2 \pm 11.0$ & & $322.0 \pm 5.8$ \\
\hline NGC 7626 & $275.1 \pm 5.2$ & 1 & $262.4 \pm 18.8$ & $299.5 \pm 11.9$ & $288.9 \pm 10.1$ & & $275.1 \pm 5.2$ \\
\hline NGC 7640 & $\cdots$ & $\ldots$ & $\cdots$ & $\cdots$ & $\cdots$ & $1,3,8$ & $48.1 \pm 22.5$ \\
\hline NGC 7741 & $\cdots$ & $\cdots$ & $\cdots$ & $29.4 \pm 8.9$ & $29.4 \pm 8.9$ & $7,8,12$ & $29.4 \pm 8.9$ \\
\hline NGC 7742 & $94.8 \pm 11$ & 1 & $\cdots$ & $73.3 \pm 8.8$ & $73.3 \pm 8.8$ & 8 & $73.3 \pm 8.8$ \\
\hline NGC 7743 & $83.8 \pm 9.3$ & 1 & $\cdots$ & $89.3 \pm 9.1$ & $89.3 \pm 9.1$ & 8 & $89.3 \pm 9.1$ \\
\hline NGC 7798 & $\cdots$ & $\cdots$ & $\cdots$ & $75.1 \pm 9.2$ & $75.1 \pm 9.2$ & 8 & $75.1 \pm 9.2$ \\
\hline NGC 7814 & $172.3 \pm 7.7$ & 1 & $191.9 \pm 14.6$ & $161.4 \pm 9.5$ & $170.5 \pm 8.0$ & & $172.3 \pm 7.7$ \\
\hline NGC 7817 & $\cdots$ & $\cdots$ & $\cdots$ & $66.7 \pm 8.4$ & $66.7 \pm 8.4$ & 6 & $66.7 \pm 8.4$ \\
\hline UGC 3714 & $\cdots$ & $\cdots$ & $\cdots$ & $104.0 \pm 9.4$ & $104.0 \pm 9.4$ & 6 & $104.0 \pm 9.4$ \\
\hline UGC 3828 & $102.3 \pm 25.1$ & 1 & $\ldots$ & $73.9 \pm 9.4$ & $73.9 \pm 9.4$ & 6 & $73.9 \pm 9.4$ \\
\hline UGC 4028 & $\cdots$ & $\cdots$ & $\cdots$ & $80.5 \pm 9.3$ & $80.5 \pm 9.3$ & 6 & $80.5 \pm 9.3$ \\
\hline UGC 6484 & $\cdots$ & $\cdots$ & $\cdots$ & $61.1 \pm 9.0$ & $61.1 \pm 9.0$ & 6 & $61.1 \pm 9.0$ \\
\hline
\end{tabular}

Notes. (1) The adopted velocity dispersion was estimated from the [N II] $\lambda 6583$ emission line following the procedure of Ho (2009). (2) Stellar features too weak in the blue or red. (3) Spectrum partly corrupted. (4) The HyperLeda value of $\sigma=240 \mathrm{~km} \mathrm{~s}^{-1}$ pertains to the "southeast-northwest component of this merging galaxy; the correct dispersion for the primary nucleus is $\sigma=100 \pm 25 \mathrm{~km} \mathrm{~s}^{-1}$ (see Section 3.4). (5) Blue spectrum not available. (6) Blue spectrum not well resolved. (7) Red spectrum not well resolved. (8) Blue fit unreliable. (9) Red fit unreliable. (10) G band corrupted in blue; masked out. (11) The literature value corresponds to the central nuclear star cluster; the Palomar value is more representative of the central $2^{\prime \prime} \times 4^{\prime \prime}$ region. (12) Velocity dispersion possibly overestimated slightly.

References. 1. HyperLeda; 2. Barth et al. (2002); 3. Kormendy \& McClure (1993); 4. Ganda et al. (2006); 5. Wegner et al. (2003); 6. Batcheldor et al. (2005); 7. Héraudeau \& Simien (1998); 8. Nelson et al. (2004); 9. Funes et al. (2002); 10. Sarzi et al. (2002); 11. Marconi et al. (2003); 12. Corsini et al. (2003); 13. Filippenko \& Ho (2003).

(This table is also available in a machine-readable form in the online journal.)

the adopted value. Most of the literature values come from the HyperLeda database (Paturel et al. 2003), which, for any given galaxy, attempts to homogenize all published measurements into a single value by applying scaling factors determined from a set of "standard" galaxies measured through a roughly constant aperture size of $2^{\prime \prime} \times 4^{\prime \prime}$. This aperture size, fortunately, exactly matches that employed in the Palomar survey.

For the final, adopted dispersion, there are strong reasons to prefer the Palomar measurements because of their homogeneity. Although in many cases their error bars formally exceed those of the literature sources, we believe that the error budget for the
Palomar measurements is realistic, as evidenced, for example, from comparison with the high-accuracy measurements from Barth et al. (2002) for galaxies in common. Nevertheless, for concreteness, the final column of Table 1 lists either the final Palomar dispersion or the literature value, if available, based on whichever has the smaller formal error bar.

In total, our catalog gives new stellar velocity dispersion measurements for 428 galaxies, $88 \%$ of the parent survey. Of these, $142(30 \%)$ have no previously published measurements. Not surprisingly, most of the new measurements are for latetype galaxies, systems where velocity dispersions are more 
challenging to obtain because of their characteristically lower values $\left(\lesssim 100 \mathrm{~km} \mathrm{~s}^{-1}\right)$ and complications due to their composite stellar populations and contamination by emission lines. Our new measurements also provide updates to a number of literature dispersions that previously had large uncertainties or, in some instances, were grossly in error.

Stellar velocity dispersions could not be derived for 58 galaxies, mostly because their stellar features are too weak. For the sake of completeness, for the 34 of these objects that have emission lines, and for which no reliable dispersions exist in the literature, we list an indirect estimate of their stellar velocity dispersion based on their observed gaseous velocity dispersion derived from the line profile of $[\mathrm{N}$ II] $\lambda 6583$. Using the current database, Ho (2009) finds that the kinematics of the ionized gas in the central few hundred parsecs of bulges generally trace the kinematics of the stars, such that $\sigma_{g} \approx(0.8-1.2) \sigma_{*}$. In detail, the normalization of the $\sigma_{g}-\sigma_{*}$ relation shows a slight dependence on nuclear $(\mathrm{H} \alpha)$ luminosity and Eddington ratio, but only for sources spectroscopically classified as AGNs (LINERs, transition objects, and Seyferts). Those classified as H II (star-forming) nuclei obey $\sigma_{g}=0.83 \sigma_{*}$ with an rms scatter of $0.19 \mathrm{dex}$. This is the relation that we use because all of the 34 emission-line sources with very weak stellar features are $\mathrm{H}$ II nuclei (Ho et al. 1997a). ${ }^{7}$ The error bars in the adopted dispersions come from the quadrature sum of the uncertainties in the original [N II] line widths (we conservatively assume $10 \%$; Ho et al. 1997a) and the 0.19 dex scatter in the $\sigma_{g}-\sigma_{*}$ relation.

\section{SUMMARY}

The Palomar spectroscopic survey has furnished considerable insights into the nature of nuclear activity in nearby galaxies (see Ho (2008) for a review). Aside from some considerations of the central stellar populations (Ho et al. 2003; Zhang et al. 2008), however, comparatively little analysis has been done on the absorption-line component of the spectra. This paper utilizes the survey spectra to derive a homogeneous set of new central stellar velocity dispersion measurements. A major obstacle is that the original survey data were not taken with this application in mind. In particular, neither the number nor the range of calibration template stars is ideally suited for deriving stellar velocity dispersions for galaxies with a wide range of composite stellar populations. The wavelength coverage of the blue-side and red-side spectra is nonstandard for velocity dispersion work and is rather sensitive to template mismatch. Moreover, the spectral resolution of the blue-side spectra is too coarse to yield reliable dispersions for most of the later-type galaxies in the sample.

We describe an effective strategy to address these challenges. We use the extensive Coudé-feed spectral library of Valdes et al. (2004) as the primary source of stellar templates. Applying a simple correction for the nominal relative resolution difference between the Valdes and Palomar systems yields velocity dispersions that show reasonably good agreement with literature values. The direct-pixel fitting code of Greene \& Ho (2006) was adapted to solve for an optimally weighted linear combination of template stars, a crucial step to match the composite stellar population typically found in later-type galaxies. We demonstrate that the $\mathrm{Ca}+\mathrm{Fe} \lambda 6495$ feature in the red-side spectra can be used to derive robust velocity dispersions, a crucial consideration because the resolution of the red setup, significantly higher than

\footnotetext{
7 In detail, Ho (2009) notes that $\sigma_{g} / \sigma_{*}$ for $\mathrm{H}$ II nuclei depends on $\sigma_{*}$, but for our present purposes we neglect this complication.
}

that of the blue setup, is sufficient to probe even the late-type systems in the survey.

Our final catalog lists a uniform set of new stellar velocity dispersions for 428 galaxies in the Palomar survey. A significant fraction of the galaxies, especially later-type systems, have no previously published velocity dispersions. Together with indirect estimates for another 34 objects and supplementary data from the literature, essentially all (482/486) of the galaxies in the Palomar survey now have central velocity dispersion measurements. The Palomar galaxies have been and continue to be heavily investigated for a variety of scientific applications. The catalog of velocity dispersions presented in this paper will add an important new dimension to the already rich database available for this much-studied galaxy sample.

We are very grateful to the staff of Palomar Observatory for their assistance with the observations over many years. We thank Aaron Barth and Dan Kelson for advice on methods of measuring velocity dispersions. An anonymous referee offered many helpful and critical comments that improved the paper. We acknowledge the usage of the HyperLeda database (http://leda.univ-lyon1.fr). The research of L.C.H. was supported by the Carnegie Institution of Washington. Support for J.E.G. was provided by NASA through Hubble Fellowship grant HF-01196, awarded by the Space Telescope Science Institute, which is operated by the Association of Universities for Research in Astronomy, Inc., for NASA, under contract NAS 526555. A.V.F. and W.L.W.S. are grateful for the financial support of the NSF, through grants AST-0607485 and AST-0606868, respectively.

\section{REFERENCES}

Barth, A. J., Ho, L. C., \& Sargent, W. L. W. 2002, AJ, 124, 2607

Batcheldor, D., et al. 2005, ApJS, 160, 76

Bender, R. 1990, A\&A, 229, 441

Bernardi, M., et al. 2003, AJ, 125, 1817

Burbidge, E. M., Burbidge, G. R., \& Fish, R. A. 1961, ApJ, 133, 393

Cappellari, M., \& Emsellem, E. 2004, PASP, 116, 138

Corsini, E. M., Pizzella, A., Coccato, L., \& Bertola, F. 2003, A\&A, 408, 873

Davies, R. L., Burstein, D., Dressler, A., Faber, S. M., Lynden-Bell, D., Terlevich, R. J., \& Wegner, G. 1987, ApJS, 64, 581

de Vaucouleurs, G., de Vaucouleurs, A., Corwin, Jr., H. G., Buta, R. J., Paturel, G., \& Fouqué, R. 1991, Third Reference Catalogue of Bright Galaxies (New York: Springer)

Filippenko, A. V., \& Ho, L. C. 2003, ApJ, 588, L13

Filippenko, A. V., Matheson, T., \& Barth, A. J. 1995, ApJ, 455, L139

Filippenko, A. V., Matheson, T., Leonard, D. C., Barth, A. J., \& Van Dyk, S. D. 1997, PASP, 109, 461

Filippenko, A. V., \& Sargent, W. L. W. 1985, ApJS, 57, 503

Funes, J. G., Corsini, E. M., Cappellari, M., Pizzella, A., Vega Beltrán, J. C., \& Bertola, F. 2002, A\&A, 388, 50

Ganda, K., Falcón-Barroso, J., Peletier, R. F., Cappellari, M., Emsellem, E., McDermid, R. M., de Zeeuw, P. T., \& Carollo, C. M. 2006, MNRAS, 367, 46

Graves, G. J., Faber, S. M., Schiavon, R. P., \& Yan, R. 2007, ApJ, 671, 243

Greene, J. E., \& Ho, L. C. 2006, ApJ, 641, 117

Héraudeau, Ph., \& Simien, F. 1998, A\&AS, 133, 317

Ho, L. C. 2008, ARA\&A, 46, 475

Ho, L. C. 2009, ApJ, 699, 638

Ho, L. C., Filippenko, A. V., \& Sargent, W. L. W. 1995, ApJS, 98, 477

Ho, L. C., Filippenko, A. V., \& Sargent, W. L. W. 1997a, ApJS, 112, 315

Ho, L. C., Filippenko, A. V., \& Sargent, W. L. W. 1997b, ApJ, 487, 568

Ho, L. C., Filippenko, A. V., \& Sargent, W. L. W. 1997c, ApJ, 487, 579

Ho, L. C., Filippenko, A. V., \& Sargent, W. L. W. 1997d, ApJ, 487, 591

Ho, L. C., Filippenko, A. V., \& Sargent, W. L. W. 2003, ApJ, 583, 159

Ho, L. C., Filippenko, A. V., Sargent, W. L. W., \& Peng, C. Y. 1997e, ApJS, 112,391

Kelson, D. D., Illingworth, G. D., van Dokkum, P. G., \& Franx, M. 2000, ApJ, 531,159 
Kormendy, J., \& McClure, R. D. 1993, AJ, 105, 1793

Marconi, A., et al. 2003, ApJ, 586, 868

McElroy, D. B. 1995, ApJS, 100, 105

Minkowski, R. 1962, in IAU Symp. 15, Problems of Extra-Galactic Research, ed. G. C. McVittie (New York: Macmillan), 112

Morton, D. C., \& Chevalier, R. 1972, ApJ, 174, 489

Nelson, C. H., Green, R. F., Bower, G., Gebhardt, K., \& Weistrop, D. 2004, ApJ, 615,652

Oke, J. B., \& Gunn, J. E. 1982, PASP, 94, 586

Paturel, G., Petit, C., Prugniel, Ph., Theureau, G., Rousseau, J., Brouty, M., Dubois, P., \& Cambrésy, L. 2003, A\&A, 412, 45

Prochaska, L. C., Rose, J. A., \& Schiavon, R. P. 2005, AJ, 130, 2666

Prugniel, Ph., Zasov, A., Busarello, G., \& Simien, F. 1998, A\&AS, 127, 117

Richstone, D. O., \& Sargent, W. L. W. 1972, ApJ, 176, 91

Rix, H.-W., \& White, S. D. M. 1992, MNRAS, 254, 389

Sandage, A. R., \& Tammann, G. A. 1981, A Revised Shapley-Ames Catalog of Bright Galaxies (Washington, DC: Carnegie Inst. of Washington)
Sargent, W. L. W., Schechter, P. L., Boksenberg, A., \& Shortridge, K. 1977, ApJ, 212,326

Sarzi, M., et al. 2002, ApJ, 567, 237

Simkin, S. M. 1974, A\&A, 31, 129

Stanford, S. A., \& Balcells, M. 1990a, in IAU Colloq. 124, Paired and Interacting Galaxies, ed. J. W. Sulentic, W. C. Keel, \& C. M. Telesco (Washington, DC:

NASA Conference Publications No. 3098), 347

Stanford, S. A., \& Balcells, M. 1990b, ApJ, 355, 59

Statler, T. S. 1995, AJ, 109, 1371

Tonry, J., \& Davis, M. 1979, AJ, 84, 1511

Valdes, F., Gupta, R., Rose, J. A., Singh, H. P., \& Bell, D. J. 2004, ApJS, 152, 251

van der Marel, R. P. 1994, ApJ, 270, 271

van der Marel, R. P., \& Franx, M. 1993, ApJ, 407, 525

Wegner, G., et al. 2003, AJ, 126, 2268

Whitmore, B. C., McElroy, D. B., \& Tonry, J. L. 1985, ApJS, 59, 1

Zhang, Y., Gu, Q.-S., \& Ho, L. C. 2008, A\&A, 487, 177 